\title{
Budgeting Costs and Returns for Central Florida Citrus Production, 2002-03
}

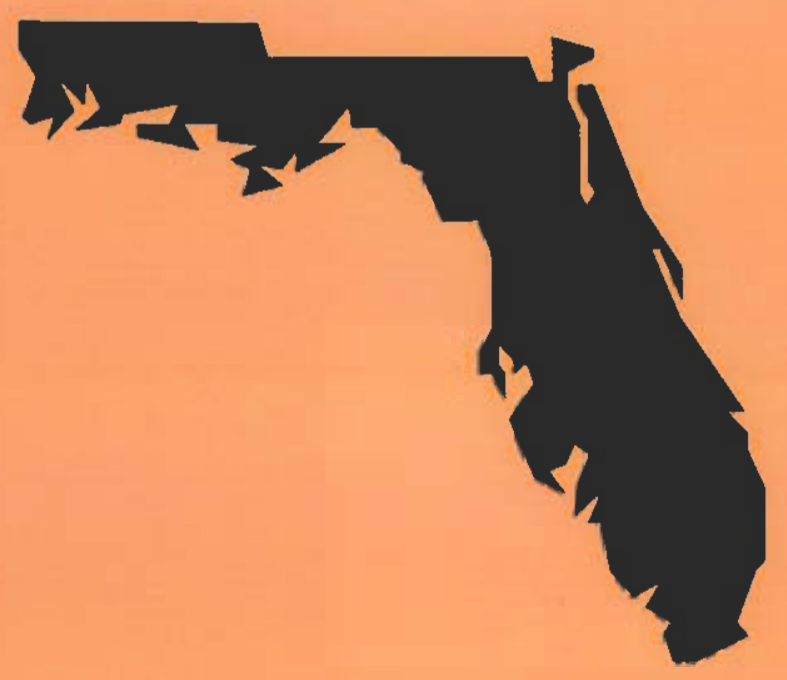

7. UNIVERSITY OF - FLORIDA

\section{IFAS EXTENSION}

Institute of Food and Agricultural Sciences

Food and Resource Econsasics Departmeat

Florida Agricultural Experiment Statisa

Florida Cooperative Extension Service

September 2003

Gainesville, FL 32611 


\section{ABSTRACT}

Estimated costs and returns of growing round oranges in the Central Florida citrus area are presented for the twentieth consecutive year. Due to the freezes of the 1980's, the Central Florida citrus area refers primarily to Polk and Highlands counties. The format presented may be used by individual growers to budget costs and returns, utilizing individual data on specific groves.

Key words: citrus, Central Florida, budgeting, costs and returns.

NOTE: The Central Florida production area refers to Polk and Highlands counties. However, the costs presented in this report are applicable to other counties such as Hardee, Hillsborough, Lake and Orange counties.

The budgeted cost information presented herein is the most current available. The budget cost items have been revised to reflect current grove practices being used by grow ers-e.g., chemical mowing, different spray materials and rates of fertilization, microsprinkler irrigation, more reset trees, etc. Thus, the 2002-03 budget costs reflect lower fertilizer and pesticide materials costs and increased per acre yield due to higher per acre tree densities.

The budget costs in this report represent a custom-managed operation. Therefore, all equipment costs are based on the average custom rate costs, and a 10 percent handling and supervision charge is add ed to the material cost.

Although the estimated annual per acre grove costs listed are representative for a mature citrus grove (10+ years old), the grove care costs for a specific grove site may differ depending upon the tree age, tree density and the grove practices performed; e.g., spot herbicide for grass/brush regrowth under trees could add an additional $\$ 19.23$ per acre; Diaprepes control could add $\$ 69.54$ per acre for soil biological control application and $\$ 73.20$ per acre for each foliar application; extensive tre e loss due to blight or tristeza could substantially increase the tree replacement and care costs; spray applications to control citrus leafminer and nematicide applications such as Temik ( $\$ 128.11$ acre) could increase the total cultural costs per acre above the average costs shown in the comparative budgets; travel and set-up costs may vary due to size of the citrus grove and distan ce from grove equipm ent barn and could add $\$ 25.98$ per acre; etc.

\section{ACKNOWLEDGEMENTS}

Appreciation is exten ded to Mrs. Jane Wilson for typing the final draft of this manusc ript.

Appreciation is also extended to the citrus growers and production managers of the Central Florida citrus production area who provided su ggestions for the revision of this manuscript. 


\section{TABLE OF CONTENTS}

$\underline{\text { Page }}$

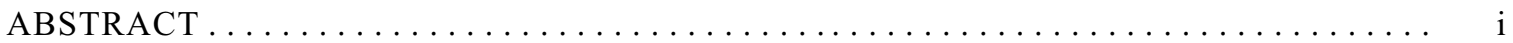

ACKNOWLEDGEMENTS $\ldots \ldots \ldots \ldots \ldots \ldots \ldots \ldots \ldots \ldots \ldots \ldots \ldots$

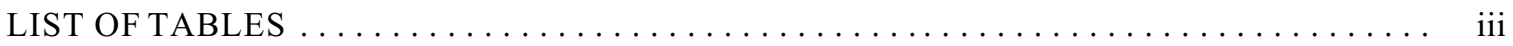

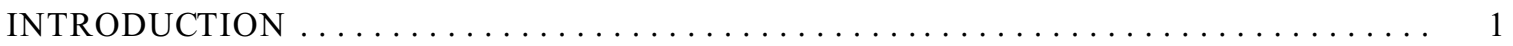

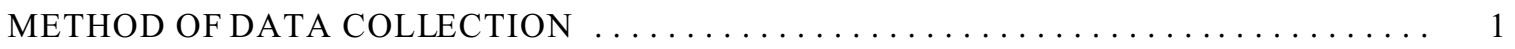

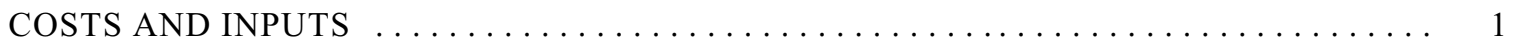

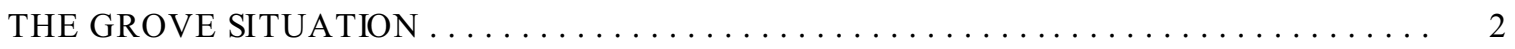

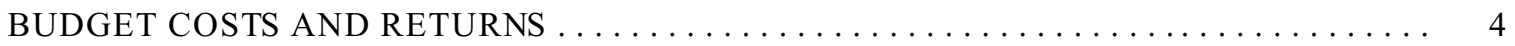

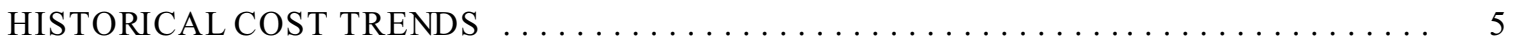

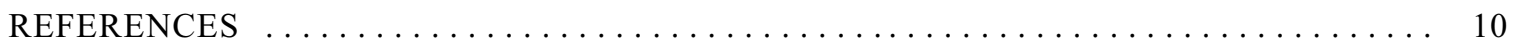

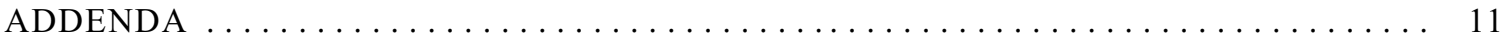

NOTE: The ADDEND A include a Listing of Grove Care Programs for Central Florida Citrus Production for Both Round Oranges and Grapefruit; 2003 custom rate summary report; cost of establishing a citrus grove; etc. Page 11 is a list of the tables included in the ADDENDA. 


\section{LIST OF TABLES}

$\underline{\text { Table }}$

$\underline{\text { Page }}$

1 Schedule of production practices and budget items for a Central Florida citrus grove, 2002-03

2 Calculation of normal production per acre, $2002-03 \ldots \ldots \ldots \ldots \ldots \ldots \ldots \ldots$

3 Estimated annual per acre costs and returns for a mature, Valencia orange grove producing for the processed market, Central Florida area, 2002-03 . . . . . . . . . 6

4 Estimated total delivered-in cost for Central Florida (Ridge) Valencia oranges grown for the processed juice market under three cultural cost programs, 2002-03 . . . . . . .

5 Estimated annual per acre costs and returns and 5-year average costs and returns for a mature, Valencia orange grove producing citrus for processing in the Central Florida

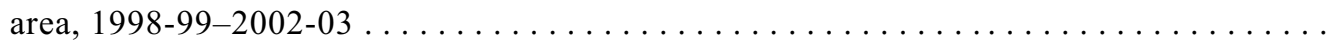

$6 \quad$ Estimated annual per acre costs and returns and 5-year average costs and returns (adjusted to 2003 dollars) for a mature, Valencia orange grove producing citrus for processing in the Central Florida area, 1998-99-2002-03 


\title{
BUDGETING COSTS AND RETURNS FOR CENTRAL FLORIDA CITRUS PRODUCTION, 2002-03
}

\author{
Ronald P. Muraro and W. C. Oswalt
}

\section{INTRODUCTION}

Budget analysis provides the basis for many grower decisions. Budget analysis can be used to calculate potential profits from an operation, to determine cash requirements for an operation, and to determine break-even prices. This report presents a budget constructed from current data and serves as a format for growers to analyze costs and returns from their individual records. During the 1980 's, several freezes occurred which changed the character of the Central Florida citrus production area. The December 1983 and January 1985 freezes caused extensive tree and acreage losses in the north central counties such as Lake and Orange counties. The December 1989 freeze resulted in severe tree damage and tree loss in North and Central Polk County. Thus, Central Florida in this report refers primarily to Polk and Highlands counties.

\section{METHOD OF DATA COLLECTION}

The data presented here were developed by surveying custom operators, input suppliers, growers, colleagues at the Citrus Research and Education Center in Lake Alfred, and County Extension Citrus Agents in the Central Florida production region. The survey is conducted annually in February and March.

\section{COSTS AND INPUTS}

Costs for various production inputs are those collected from citrus growers as well as the average of the data obtained from annual custom rate, chemical, and fertilizer surveys. Growers' costs are shown in the ADDENDA, Table 1-A through 7-A. The custom rate costs are shown in Table 8-A and the various chemical and fertilizer costs are shown in Table 9-A and 10-A in the ADDENDA. The budget costs represent a custom-manage d operation. Therefore, all equipment costs are based upon the

RONALD P. MURARO is a Professor of Food and Resource Economics and Extension Farm Management Economist stationed at the Citrus Research and Education Center, Lake Alfred. W. C. OSWALT is a Multi-county Extension Agent, Citrus in Polk and Hillsborough Counties, Bartow. 
average custom-rate costs and a 10 percent handling and supervision charge is added to the material cost.

Although brand names are used in many of the tables in the ADDENDA, this does not imply endorsement by the University of Florida. It is mere ly an attempt to depict typical production practices.

All tables have a column reserved for the individual growers to insert data from a particular grove. This will allow a comparison of the grower's costs with those presented.

\section{THE GROVE SITUATION}

Production practices for a Central Florida round orange grove are shown in Table 1 with times during the year when they would likely be performed. There are two benefits to developing such a table for an individual grove. First, it shows what work is needed and when, so that operations can be planned well in advance. Second, it can be helpful if an annual cash flow analysis is developed to plan financing. The individual grower may benefit from developing a plan for a particular grove.

Specific production practices vary from grove to grove making it difficult to define a "typical" grove. Many combinations of practices and various tree variety combinations produce acceptable yields and returns. Although the example represents a Valencia orange grove, the cost and return data are designed to be applicable to most grove situations. A grower, realtor, or land appraiser can substitu te individual grove costs and expected returns into the budget format and develop a budget for a particular grove. A "your cost" column is appropriately provided for this purpose in subsequent tables.

In the following budget, above average management and cultural practices are assumed. Beyond this general assumption, the following specifics are assumed.

1. A $10+$ year-old, low volume-irrigated grove;

2. Variety is Valencia round orange;

3. Tree loss is 3 percent an nually;

4. Trees are pulled and replaced when production falls below 50 percent of expected yield;

5. Production is for processed use;

6. Tree density is 112 trees per acre; and

7. Custom-ca retaker is providing grove manage ment. 


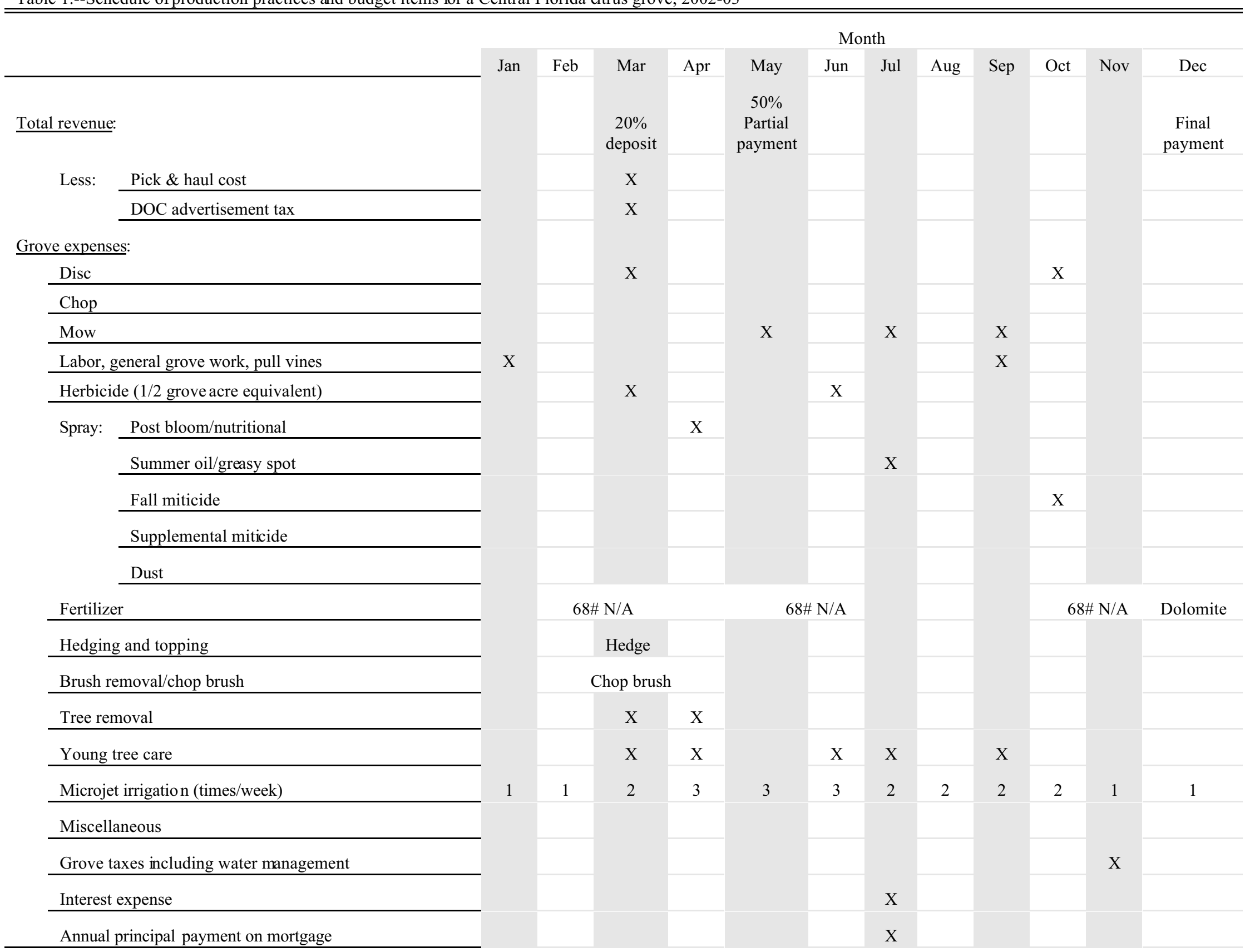

${ }^{a}$ This is a suggested schedule of practices. Actual practices would not necessarily be done on the exact schedule shown here. 
As a result of tree losses and replac ement, the tre e ages will vary. The budgetreflects the following age distribution:

$\underline{\text { Situation }}$

$\begin{array}{rl}3 \% & \text { pulled and reset } \\ 3 \% & 1 \text { year old } \\ 3 \% & 2 \text { years old } \\ 3 \% & 3 \text { years old } \\ 3 \% & 4 \text { years old } \\ 45 \% & 5-19 \text { years old } \\ 3 \% & \text { producing } 50 \% \text { of expected yield } \\ 37 \% & \text { mature producing }\end{array}$

Yield $\underline{\text { boxes/tree }}$

0.0

0.0

0.0

0.6

0.9

3.8

2.8

5.5

Calculation of normal production per acre is shown in Table 2. Note that the proportion-of-treesby-age column only adds to 91 percent since 9 percent of the trees are non-bearing.

Table 2.--Calculation of normal production per acre, 2002-03

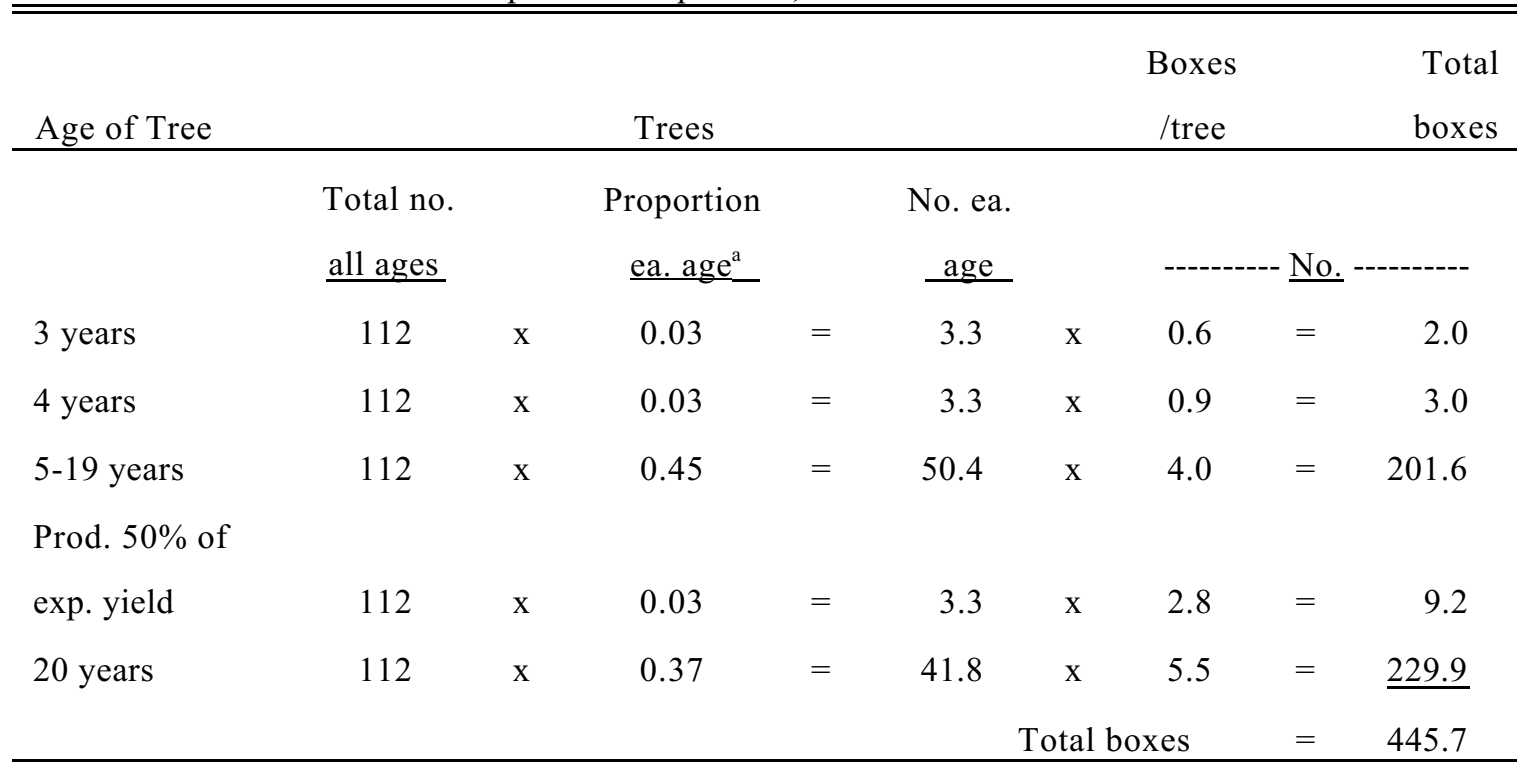

${ }^{\text {aP }}$ roportion adds up to 0.91 (91 percent) as 9 percent of the trees were non-bearing (pulled and reset, 1 and 2 year old trees).

\section{BUDGET COSTS AND RETURNS}

The estimated budget costs and retums for the Central Florida grove situation are show $\mathrm{n}$ in Table 3. The bud geted costs represent one possible citrus production program and were selected from the costs shown in the ADDENDA tables. The gross revenue estimates are based on the projected yields in Table 3 and estimated preliminary on-tree prices for the 2002-03 season. Grove establishment and reset costs, harvesting and packing charges can be found in Tables 11-A through 15-A in the ADDENDA. 
Also, historical on-tree prices for selected Florida citrus varieties are shown in Table 16-A of the ADDENDA.

As shown in Table 3, the total revenue for process ed-market Valencia oranges is estimated to be $\$ 2,105.12$ per acre. Total specified costs are $\$ 825.59$ and are comprised of grove care costs of $\$ 777.69$, plus management cost of $\$ 48.00$. Return to land and trees of $\$ 1,279.53$ represents net return above variable costs. At 300 and 500 boxes per acre, respectively, the break-even price requir ed to cover grove care costs for Valencia oranges range from $\$ 2.59$ to $\$ 1.56$ per box on-tree and $\$ 0.72$ to $\$ 0.56$ per pounds solids delivered-in.

Ad valorem taxe s, and overh ead and a dministrative costs (such a s water drain age district taxes, crop insurance, and other grower asse ssments) can add up to 12 percent to the total grove care costs. These costs vary from grove to grove depending on age, location, variety of fruit, etc. and should be considered in arriving at net return to land, trees and ownership (total return minus total costs). Harvest costs (pick, roadside, and hauling costs) also add to the total fruit cost delivered to either a processing plant or fresh fruit packinghouse. Also, average annual debt payment (principal and interest) may be as high as $\$ 440$ per acre $(\$ 3,750$ average debt per acre @10 percent interest amortized over 20 years $)$ which would reduce total available cash for grove expans ion or other investment.

An estimated "delivered-in" c osts are shown for process ed orang es in Table 4. "Delivere d-in" costs include grove care costs (Table 3) plus harvesting, regulatory, and grower assessment costs. The “delivered-in" cost is presented as a cost per acre, per box, and per pound solids. Three possible budget cost scenarios are presented(Refer to Table 13-A): 1) Low Cost Processed Cultural Program; 2)Reduced Cost Cultural Program; and 3) Typical/Historical Cultural Program. Scen arios 1 and 2 represent costs of two possible cultural programs directed toward reducing the expenditures for fruit grown primarily for the processed market. The third scenario represents typical costs of grove practices which have been performed for citrus grown for the fresh/processed fruit market. Modified herbicide and/or spray and fertilizer programs account for the reduced costs. NOTE: Before modifying a grove management program to reduce costs, an evaluation of the market program (processed or fresh), yield, and specific cultural problems (nutrition, disease, etc.) for the specific grove site should be made.

\section{HISTORICAL COST TRENDS}

Annual budgets of costs and returns for mature, processed Valencia oranges in the Central Florida area have been developed and published the past four years. Estimated cost and return histories for 1998-99 through 2001-02 along with 2002-03, and a five-year average are presented in Table 5. To allow comparisons in current values, these same costs and returns, adjusted to 2003 dollars, are presented in Table 6. 


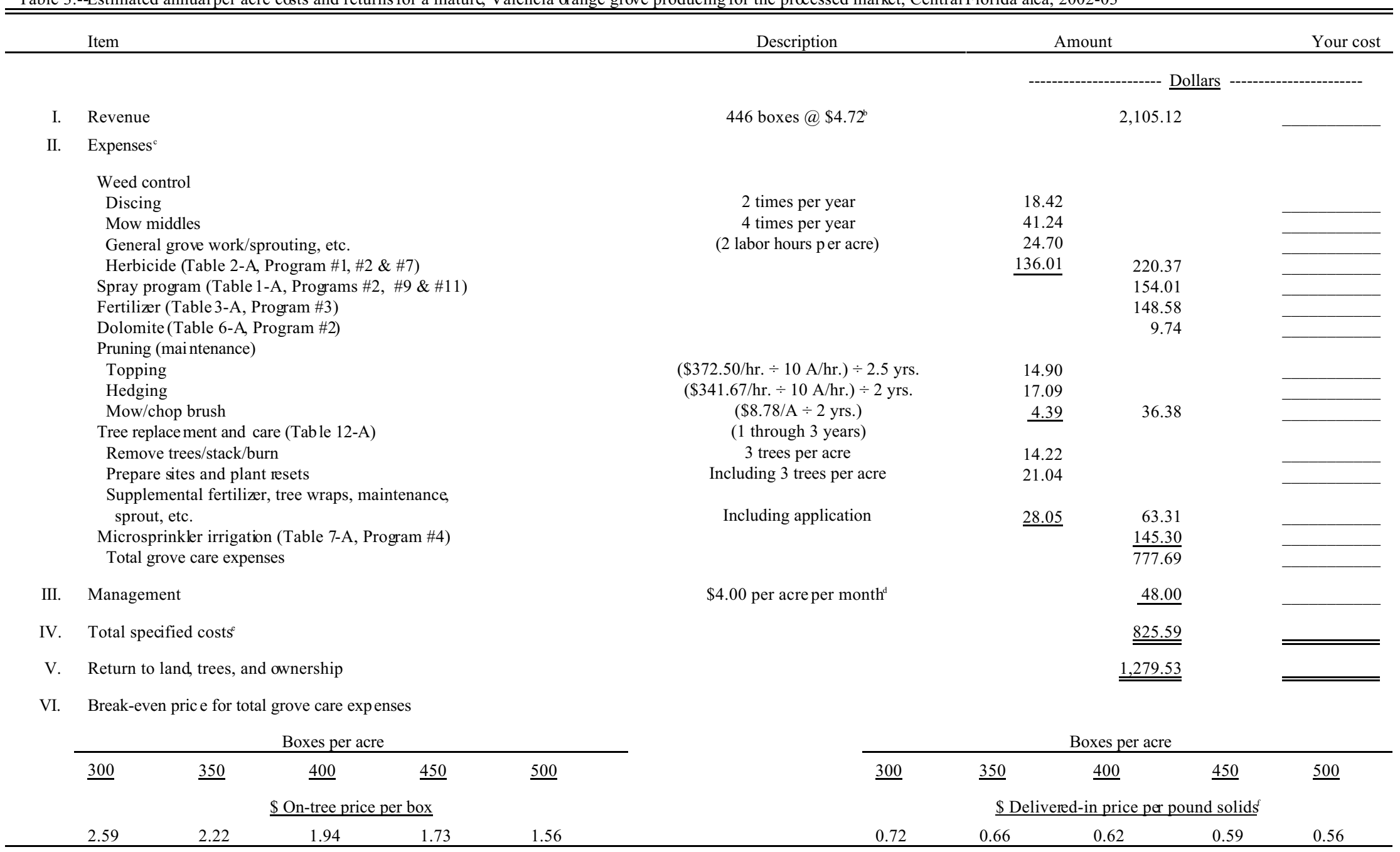

${ }^{a}$ Although the estimated annual per acre grove costs shown in Table 3 are representative for a mature Central Florida Valencia orange grove, the grove care costs for a specific grove site may differ depending upon the grove practices performed; e.g., a Temik application would add $\$ 128.11$ per acre; extensive tree loss due to blight or tristeza would double the tree replacement and care costs; travel and set-up costs may vary due to size of citrus grove and distance from grove equipment bam.

${ }^{\mathrm{b}}$ On-tree price per box is preliminary; assumes price for processed oranges only.

'Assumes material custom applied; therefore, a 10 percent handling and supervision charge is added to material cost.

${ }^{d}$ Other methods to estimate a management cost--e.g, $5 \%$ of gross revenue or $10 \%$ of total grove care costs-are used in the industry. Other selected methods will give a different return to land and trees than reported here.

'Other cost items which are not included in the budget are ad valorem taxes and interest on grove investment. In addition to these cost items, overhead and administrative costs, such as water drainage/district ta xes, crop insu rance, and ot her grower assessments, can add up to 12 percent to the total grove care costs. These costs vary from grove to grove depending on a ge, location, and time of purchase or grove establishment.

${ }^{\mathrm{f}}$ Assumes 6.7 pounds solids per box and $\$ 2.19$ pick and haul cost per box (including canker decontamina tion costs) and Department of Citrus ad vertising assessment of $\$ 0.165$ per box. 
Table 4.--Estimated total delivered-in cost for Central Florida (Ridge) Valencia oranges grown for the processed market under three cultural cost programs, 2002-03

\begin{tabular}{|c|c|c|c|c|c|c|c|c|c|}
\hline \multirow[t]{2}{*}{$\begin{array}{l}\text { Represents a mature }(10+\text { years old) } \\
\text { Central Florida (Ridge) Orange Grove }\end{array}$} & \multicolumn{3}{|c|}{$\begin{array}{c}\text { Processed Valencia Oranges } \\
\text { Low Cost } \\
\text { Cultural Program }\end{array}$} & \multicolumn{3}{|c|}{$\begin{array}{c}\text { Processed Valencia Oranges } \\
\text { Reduced Cost } \\
\text { Cultural Program }\end{array}$} & \multicolumn{3}{|c|}{$\begin{array}{c}\text { Fresh/Processed Valencia Oranges } \\
\text { Cultural Program }\end{array}$} \\
\hline & $\$ /$ Acre & $\$ /$ Box & \$/P.S. & $\$ /$ Acre & $\$ /$ Box & $\$ / P . S$. & $\$ /$ Acre & $\$ /$ Box & \$/P.S. \\
\hline Total Production/C ultural Costs & $\$ 722.78$ & $\$ 1.621$ & $\$ 0.2419$ & $\$ 777.69$ & $\$ 1.744$ & $\$ 0.2603$ & $\$ 837.24$ & $\$ 1.877$ & $\$ 0.2802$ \\
\hline Interest on Operating (Cultural) Costs & 36.14 & 0.081 & 0.0121 & 38.88 & 0.087 & 0.0130 & 41.86 & 0.094 & 0.0140 \\
\hline Manag ement Costs & 48.00 & 0.108 & 0.0161 & 48.00 & 0.108 & 0.0161 & 48.00 & 0.108 & 0.0161 \\
\hline \multicolumn{10}{|l|}{ Taxes/Regulatory Costs: } \\
\hline Property Tax and Water Management Tax & 58.92 & 0.132 & 0.0197 & 58.92 & 0.132 & 0.0197 & 58.92 & 0.132 & 0.0197 \\
\hline Canker D econtamination Costs & 4.50 & $\underline{0.010}$ & $\underline{0.0015}$ & 4.50 & $\underline{0.010}$ & $\underline{0.0015}$ & 4.50 & $\underline{0.010}$ & $\underline{0.0015}$ \\
\hline Total Dire ct Grower Costs & $\$ 870.34$ & $\$ 1.951$ & $\$ 0.2913$ & $\$ 927.99$ & $\$ 2.081$ & $\$ 0.3106$ & $\$ 990.52$ & $\$ 2.221$ & $\$ 0.3315$ \\
\hline Interest on A verage Capital Investment Costs & 377.90 & $\underline{0.847}$ & $\$ 0.1265$ & $\underline{377.90}$ & $\underline{0.847}$ & $\underline{0.1265}$ & $\underline{377.90}$ & $\underline{0.844}$ & $\underline{0.1259}$ \\
\hline Total Grower Costs & $\$ 1,248.24$ & $\$ 2.799$ & $\$ 0.4177$ & $\$ 1,305.89$ & $\$ 2.928$ & $\$ 0.4370$ & $\$ 1,368.42$ & $\$ 3.064$ & $\$ 0.4574$ \\
\hline \multicolumn{10}{|l|}{ Harvesting and Assessment Costs: } \\
\hline Canker D econtamination Cos ts & 974.51 & 2.185 & 0.3261 & 974.51 & 2.185 & 0.3261 & 974.51 & 2.185 & 0.3261 \\
\hline DOC Assessment & 73.59 & $\underline{0.165}$ & $\underline{0.0246}$ & 73.59 & $\underline{0.165}$ & $\underline{0.0246}$ & 73.59 & $\underline{0.165}$ & $\underline{0.0246}$ \\
\hline Total Harve sting and A ssessment Costs & $1,048.10$ & 2.350 & 0.3507 & $1,048.10$ & 2.350 & 0.3507 & $1,048.10$ & 2.350 & 0.3507 \\
\hline Total Delivered-In Cost & $\$ \underline{\underline{2,296.34}}$ & $\$ \underline{\underline{5.149}}$ & $\$ \underline{\underline{0.7685}}$ & $\$ \underline{\underline{2,353.99}}$ & $\$ \underline{\underline{5.278}}$ & $\$ \underline{\underline{0.7878}}$ & $\$ \underline{\underline{2,416.52}}$ & $\$ \underline{\underline{5.414}}$ & $\$ \underline{\underline{0.8081}}$ \\
\hline $\begin{array}{l}\text { P.S. = Pound Solids } \\
\text { Yield: } 446 \text { boxes/acre@6.8 P.S. per box } \\
112 \text { trees per acre }\end{array}$ & \multicolumn{3}{|c|}{$\begin{array}{l}\text { Refer to cultural program shown } \\
\text { in Table 13-A. } \\
\text { Two sum mer oil sprays with oil, } \\
\text { copper, and nutritionals. }\end{array}$} & \multicolumn{3}{|c|}{$\begin{array}{c}\text { Refer to cultural program shown } \\
\text { in Table } 3 .\end{array}$} & \multicolumn{3}{|c|}{$\begin{array}{l}\text { Refer to cultural program shown in } \\
\text { Table 13-A. } \\
\text { A Fall Miticide Spray added to the } \\
\text { cultural program shown in Table } 3 .\end{array}$} \\
\hline
\end{tabular}


Table 5.--Estimated annual per acre costs and returns and 5-year average costs and returns for a mature, Valencia orange grove producing citrus for processing in the Central Florida area, 1998-99-2002-03

\begin{tabular}{|c|c|c|c|c|c|c|}
\hline Year & $\begin{array}{r}\text { On-tree } \\
\text { price/box }\end{array}$ & Yield & $\begin{array}{c}\text { Gross } \\
\text { revenue }\end{array}$ & $\begin{array}{c}\text { Total grove } \\
\text { care expenses }\end{array}$ & $\begin{array}{c}\text { Total specified } \\
\text { costs }^{\mathrm{f}}\end{array}$ & $\begin{array}{l}\text { Net return to land, } \\
\text { trees, and ownership }\end{array}$ \\
\hline & & & ------- & ------------. & ollars --------- & ------------------- \\
\hline 1998-99 & $\$ 5.32$ & $448^{\mathrm{c}}$ & $2,383.36$ & 770.79 & 818.79 & $1,565.11$ \\
\hline 1999-00 & $\$ 4.31$ & $448^{\mathrm{c}}$ & $1,930.88$ & 783.43 & 831.43 & $1,099.45$ \\
\hline $2000-01$ & $\$ 3.70$ & $436^{\mathrm{d}}$ & $1,613.20$ & $758.85^{\mathrm{e}}$ & 806.85 & 806.35 \\
\hline 2001-02 & $\$ 3.96$ & 446 & $1,766.16$ & 767.77 & 815.77 & 950.39 \\
\hline $2002-03$ & $\$ 4.72^{\mathrm{b}}$ & 446 & $2,105.12$ & 777.69 & 825.59 & $1,279.53$ \\
\hline 5-yr. avg. & $\$ 4.40$ & 445 & $1,958.00$ & 771.74 & 819.71 & $1,138.29$ \\
\hline
\end{tabular}

${ }^{a}$ On-tree prices for processed oranges only as reported by the Florida Agricultural Statistics Service.

${ }^{b}$ Preliminary estimate by authors at time of printing and is not a published price.

${ }^{c}$ Higher per acre yield is due to increased average tree density of Valencia citrus groves in Central Florida.

${ }^{\mathrm{d}}$ The severe drought affected yields for the 2000-01 season.

${ }^{\text {e}}$ Two summer oil sprays (one with nutritionals) were used in the 2001-02 and 2002-03 budget estimates.

${ }_{\mathrm{f}}^{\mathrm{f}} \mathrm{A}$ management cost of $\$ 4.00$ per acre per month is included. Fixed costs such as taxes, debt service, and crop insurance are not included. 
Table 6.--Estimated annual per acre costs and returns and 5 -year average costs and returns (adjusted to 2003 dollars) for a mature, Valencia orange grove producing citrus for processing in the Central Florida area, 1998-99-2002-03

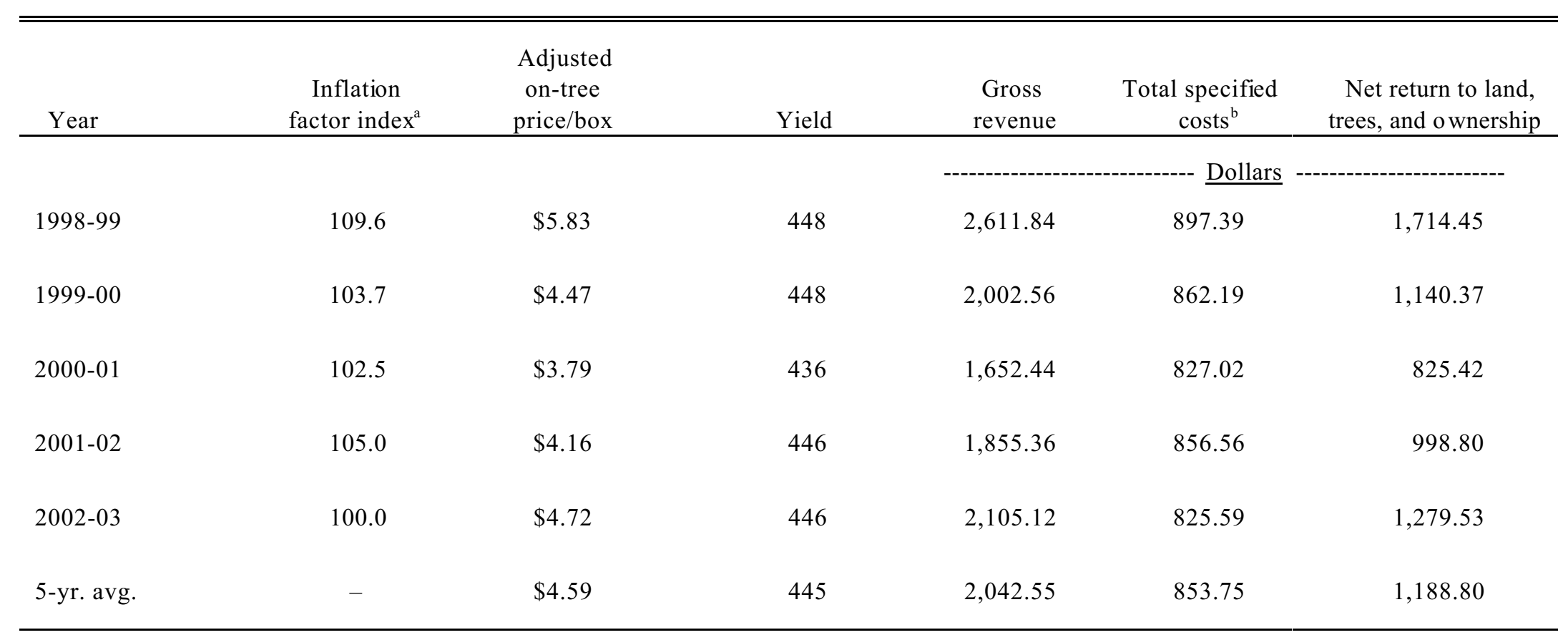

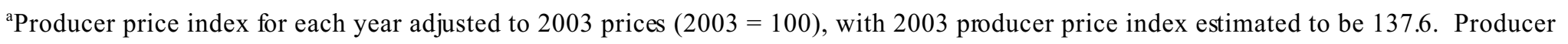
price index for other years are: $1999=125.5 ; 2000=132.7 ; 2001=134.2 ;$ and $2002=131.1$.

${ }^{\mathrm{b}} \mathrm{A}$ management cost of $\$ 4.00$ per acre per month is included. Fixed costs such as taxes, debt service, and crop insurance are not included. (Refer to Table 5.) 


\section{REFERENCES}

1. Citrus Summary 2001-02. Florida Agricultural Statistics Service. Florida Agricultural Statistics.

2. Muraro, Ronald P. "A Listing ofEstimated Comparative CentralFlorida (Ridge) Citrus Production Costs Per Acre for 2002-03." Lake Alfred Citrus Research and Education Center(CREC) Report. Lake Alfred, FL: June 2003.

3. _ "Cost for Establishing, Planting, and Maintaining a Citrus Grove through Four Years of Age, North Florida Area.” Lake Alfred CREC Report. Lake Alfred, FL: March 1990.

4. __ _Estimated Cost of Planting and Maintaining a Reset Citrus Tree through Three Years of Age." Lake Alfred CREC Report. Lake Alfred, FL: December 2000.

5. _ _ _ . "A Listing of 2003 Custom Rates Reported by Thirty Ridge Citrus Caretakers." Lake Alfred CREC Report. Lake Alfred, FL: June 2003.

6. Savage, Zach. Citrus Yields Per Tree Age. Univ. of Fla. Agr. Ext. Ser. 60-8. Gainesville: 1960.

7. Timmer, L. W.(Ed.). 2003 Florida Citrus Pest Management Guide. Univ. of Fla. Coop. Ext. Svc. SP 43. Gainesville: Jan. 2003. 150 p.

8. Tucker, D. P. H., A. K. Alva, L. K. Jackson, and T. A. Wheaton (Eds.). Nutrition of Florida Citrus Trees. Univ. of Fla. Coop. Ext. Svc. SP 169. Gainesville: 1995. 61 pp. 
ADDENDA: $\quad$ Listing of Grove Care Programs for Central Florida Citrus Production for Both Round Oranges and Grapefruit ${ }^{\mathrm{a}}$

$\underline{\text { Page }}$

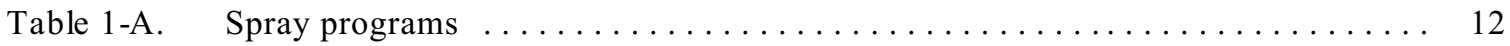

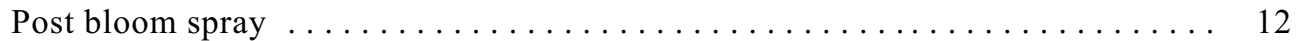

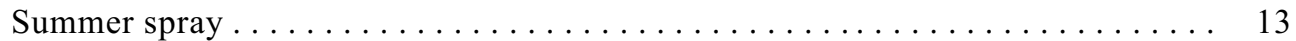

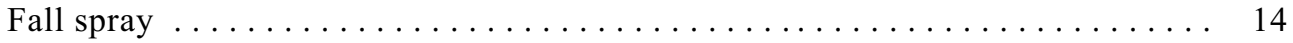

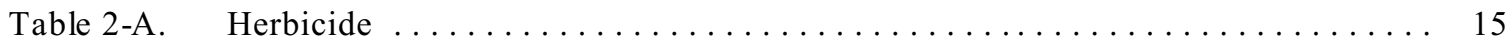

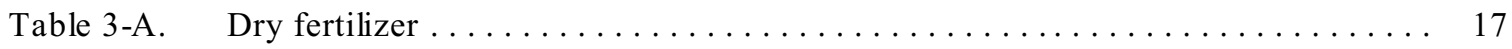

Table 4-A. $\quad$ Liquid fertilizer (Double boom application) $\ldots \ldots \ldots \ldots \ldots \ldots \ldots \ldots \ldots \ldots$

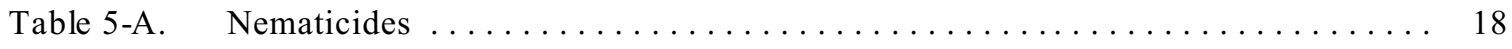

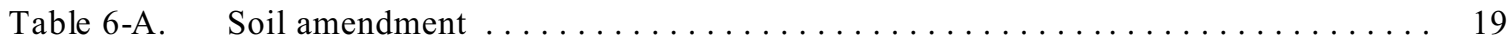

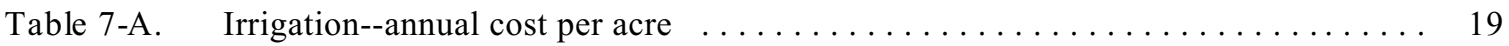

Permanent overhead ................................. 19

Microsprinkler ............................... 19

Table 8-A. A listing of 2003 custo $m$ rates reported by thirty-three Ridge citrus care takers $\ldots 20$

Table 9-A. 2003 summary of average chemical price estimates $\ldots \ldots \ldots \ldots \ldots \ldots \ldots .22$

Table 10-A. 2003 summary of average fertilizer price estimates $\ldots \ldots \ldots \ldots \ldots \ldots \ldots \ldots$

Table 11-A. Cost for establishing, planting and maintaining a citrus grove through four years

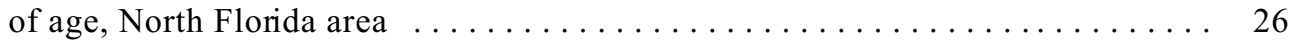

Table 12-A. Estimated cost of planting and maintaining a reset citrus tree through three years

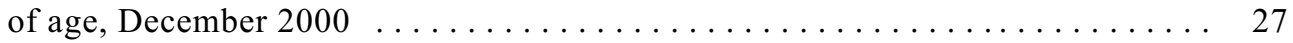

Table 13-A. A listing of estimated comparative Central Florida (Ridge) citrus production

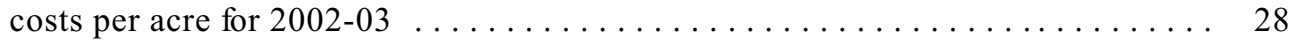

Table 14-A. Estimated average picking, roadsiding and hauling charges for Florida citrus,

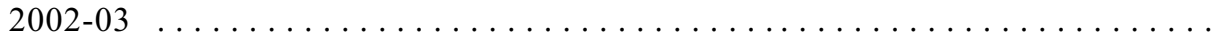

Table 15-A. Estimated average packing charges for Florida citrus, $2002-03 \ldots \ldots \ldots \ldots \ldots$

Table 16-A. Historic prices for selected citrus varieties . . . . . . . . . . . . . . 31

Table 17-A. Debt which can be supported per $\$ 1,000.00$ annual payment capacity $\ldots \ldots \ldots . \quad 32$ Abbreviations for important chemicals are:
$\mathrm{B}=$ Boron
$\mathrm{Fe}=$ Iron
$\mathrm{Mn}=$ Manganese
$\mathrm{Zn}=\mathrm{Zinc}$
$\mathrm{Cu}=$ Copper
$\mathrm{Mg}=$ Magnesium
$\mathrm{N}=$ Nitrogen

${ }^{a}$ The costs in the ADDENDA represent a custom managed operation. Therefore, all equipment costs are based upon the average custom rate costs and a 10 percent handling and supervision charge is added to the material cost. 
Table 1-A.--Spray programs

POST BLOOM SPRAY

\begin{tabular}{|c|c|c|c|c|}
\hline \multirow[t]{7}{*}{ Spray Program \#1 } & $\underline{\text { Materials/Ing redients }}$ & $\begin{array}{l}\text { Amount } \\
\text { /Acre }\end{array}$ & $\underline{\text { Cost } / \text { Acre }}$ & $\begin{array}{c}\text { Your } \\
\text { Cost/Acre } \\
\end{array}$ \\
\hline & Oil $97+\%$ & 5 gals & $\$ 12.40$ & \\
\hline & $\mathrm{Cu}(50 \%$ metallic $)$ & $10 \mathrm{lbs}$ & 14.00 & \\
\hline & $\mathrm{Zn}$ & $5 \mathrm{lbs}$ & 4.35 & \\
\hline & $\mathrm{Mn}$ & $10 \mathrm{lbs}$ & 3.40 & \\
\hline & $\begin{array}{l}\text { Ground Application } \\
\quad \text { (PTO driven airblast) }\end{array}$ & 150 gals & $\underline{25.21}$ & \\
\hline & Total per Application & & $\$ \underline{\underline{59.36}}$ & \\
\hline \multirow{7}{*}{$\begin{array}{l}\text { Spray Program \#2 } \\
\text { (Scab/melanose) }\end{array}$} & $\underline{\text { Materials/Ing redients }}$ & $\begin{array}{l}\text { Amount } \\
\text { /Acre } \\
\end{array}$ & $\underline{\text { Cost/Acre }}$ & $\begin{array}{c}\text { Your } \\
\text { Cost/Acre } \\
\end{array}$ \\
\hline & $\mathrm{Cu}(50 \%$ metallic $)$ & $10 \mathrm{lbs}$ & $\$ 14.00$ & \\
\hline & $\mathrm{Zn}$ & $5 \mathrm{lbs}$ & 4.35 & \\
\hline & & $10 \mathrm{lbs}$ & 3.40 & \\
\hline & Micromite $25 \mathrm{WP}$ & $1.25 \mathrm{lbs}$ & 42.93 & \\
\hline & $\begin{array}{l}\text { Ground Application } \\
\quad \text { (PTO driven airblast) }\end{array}$ & 150 gals & $\underline{25.21}$ & \\
\hline & Total per Application & & $\$ \underline{\underline{89.89}}$ & \\
\hline \multirow[t]{5}{*}{ Spray Program \#3 } & $\underline{\text { Materials/Ing redients }}$ & $\begin{array}{l}\text { Amount } \\
\text { /Acre } \\
\end{array}$ & $\underline{\text { Cost/Acre }}$ & $\begin{array}{c}\text { Your } \\
\text { Cost/Acre } \\
\end{array}$ \\
\hline & $\mathrm{Cu}(50 \%$ metallic $)$ & $15 \mathrm{lbs}$ & $\$ 21.00$ & \\
\hline & Ethion & $6 \mathrm{pts}$ & 27.84 & \\
\hline & $\begin{array}{l}\text { Ground Application } \\
\quad \text { (engine driven airblast) }\end{array}$ & 250 gals & $\underline{28.65}$ & \\
\hline & Total per Application & & $\$ 77.49$ & \\
\hline \multirow[t]{6}{*}{ Spray Program \#4 } & $\underline{\text { Materials/Ing redients }}$ & $\begin{array}{l}\text { Amount } \\
\text { /Acre } \\
\end{array}$ & $\underline{\text { Cost/Acre }}$ & $\begin{array}{c}\text { Your } \\
\text { Cost/Acre } \\
\end{array}$ \\
\hline & Vendex 50WP & $2 \mathrm{lbs}$ & $\$ 32.72$ & \\
\hline & $\mathrm{Zn}$ & $5 \mathrm{lbs}$ & 4.35 & \\
\hline & $\mathrm{Mn}$ & $10 \mathrm{lbs}$ & 3.40 & \\
\hline & $\begin{array}{l}\text { Ground Application } \\
\text { (PTO driven airblast) }\end{array}$ & 150 gals & 25.21 & \\
\hline & Total per Application & & $\$ 65.68$ & \\
\hline
\end{tabular}


Table 1-A.--Spray program s (cont'd.)

POST BLOOM SPRAY (cont'd.)

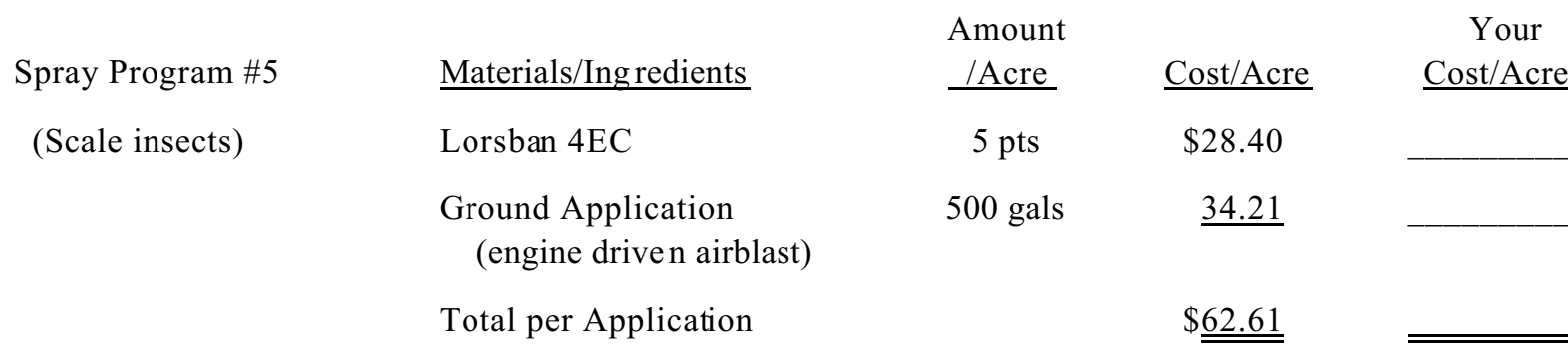

\section{$\underline{\text { SUMMER SPRAY }}$}

\begin{tabular}{|c|c|c|c|c|}
\hline \multirow[t]{6}{*}{ Spray Program \#6 } & $\underline{\text { Materials/Ing redients }}$ & $\begin{array}{l}\text { Amount } \\
\text { /Acre } \\
\end{array}$ & $\underline{\text { Cost/Acre }}$ & $\begin{array}{c}\text { Your } \\
\text { Cost/Acre } \\
\end{array}$ \\
\hline & Oil 97+\% & 5 gals & $\$ 12.40$ & \\
\hline & $\mathrm{Cu}(50 \%$ material $)$ & $7 \mathrm{lbs}$ & 9.80 & \\
\hline & Ethion & $6.0 \mathrm{pts}$ & 27.84 & \\
\hline & $\begin{array}{l}\text { Ground Application } \\
\text { (PTO driven airblast) }\end{array}$ & 250 gals & $\underline{28.80}$ & \\
\hline & Total per Application & & $\$ \underline{\underline{78.84}}$ & \\
\hline \multirow[t]{5}{*}{ Spray Program \#7 } & Materials/Ing redients & $\begin{array}{l}\text { Amount } \\
\text { /Acre } \\
\end{array}$ & $\underline{\text { Cost/Acre }}$ & $\begin{array}{c}\text { Your } \\
\text { Cost/Acre } \\
\end{array}$ \\
\hline & Oil $97+\%$ & 5 gals & $\$ 12.40$ & \\
\hline & Ethion & $5 \mathrm{pts}$ & 23.20 & \\
\hline & $\begin{array}{l}\text { Ground Application } \\
\quad \text { (engine driven airblast) }\end{array}$ & 500 gals & $\underline{34.21}$ & \\
\hline & Total per Application & & $\$ \underline{\underline{69.81}}$ & \\
\hline \multirow[t]{6}{*}{ Spray Program \#8 } & $\underline{\text { Materials/Ing redients }}$ & $\begin{array}{l}\text { Amount } \\
\text { /Acre }\end{array}$ & $\underline{\text { Cost/Acre }}$ & $\begin{array}{c}\text { Your } \\
\text { Cost/Acre } \\
\end{array}$ \\
\hline & Oil 97+\% & 5 gals & $\$ 12.40$ & \\
\hline & Agri-Mek & $10 \mathrm{ozs}$ & 51.90 & \\
\hline & $\mathrm{Cu}(50 \%$ material $)$ & $7 \mathrm{lbs}$ & 9.80 & \\
\hline & $\begin{array}{l}\text { Ground Application } \\
\quad \text { (engine driven airblast) }\end{array}$ & 250 gals & $\underline{28.65}$ & \\
\hline & Total per Application & & $\$ 102.75$ & \\
\hline
\end{tabular}


Table 1-A.--Spray program s (cont'd.)

$\underline{\text { SUMMER SPRAY (cont'd.) }}$

\begin{tabular}{|c|c|c|c|c|}
\hline \multirow[t]{6}{*}{ Spray Program \#9 } & $\underline{\text { Materials/Ing redients }}$ & $\begin{array}{l}\text { Amount } \\
\text { /Acre } \\
\end{array}$ & $\underline{\text { Cost/Acre }}$ & $\begin{array}{c}\text { Your } \\
\text { Cost/Acre } \\
\end{array}$ \\
\hline & Oil $97+\%$ & 5 gals & $\$ 12.40$ & \\
\hline & Micromite & $1.25 \mathrm{lbs}$ & 42.93 & \\
\hline & $\mathrm{Cu}(50 \%$ material $)$ & $7 \mathrm{lbs}$ & 9.80 & \\
\hline & $\begin{array}{l}\text { Ground Application } \\
\quad \text { (PTO driven airblast) }\end{array}$ & 250 gals & $\underline{28.80}$ & \\
\hline & Total per Application & & $\$ 93.93$ & \\
\hline \multirow[t]{4}{*}{ Spray Program \#10 } & $\underline{\text { Materials/Ing redients }}$ & $\begin{array}{l}\text { Amount } \\
\text { /Acre }\end{array}$ & $\underline{\text { Cost/Acre }}$ & $\begin{array}{c}\text { Your } \\
\text { Cost/Acre } \\
\end{array}$ \\
\hline & Oil $97+\%$ & 7 gals & $\$ 17.36$ & \\
\hline & $\begin{array}{l}\text { Ground Application } \\
\quad \text { (engine driven airblast) }\end{array}$ & 250 gals & $\underline{28.65}$ & \\
\hline & Total per Application & & $\$ \underline{\underline{46.01}}$ & \\
\hline \multirow[t]{8}{*}{ Spray Program \#11 } & Materials/Ing redients & $\begin{array}{l}\text { Amount } \\
\text { /Acre } \\
\end{array}$ & $\underline{\text { Cost/Acre }}$ & $\begin{array}{c}\text { Your } \\
\text { Cost/Acre } \\
\end{array}$ \\
\hline & $\mathrm{Cu}(50 \%$ metallic $)$ & $7 \mathrm{lbs}$ & $\$ 9.80$ & \\
\hline & Oil $97+\%$ & 5 gals & 12.40 & \\
\hline & $\mathrm{Zn}$ & $5 \mathrm{lbs}$ & 4.35 & \\
\hline & $\mathrm{Mn}$ & $10 \mathrm{lbs}$ & 3.40 & \\
\hline & $\mathrm{B}$ & $0.25 \mathrm{lbs}$ & 1.33 & \\
\hline & $\begin{array}{l}\text { Ground Application } \\
\quad \text { (PTO driven airblast) }\end{array}$ & 250 gals & $\underline{28.80}$ & \\
\hline & Total per Application & & $\$ \underline{\underline{60.08}}$ & \\
\hline
\end{tabular}

\section{$\underline{\text { FALL SPRAY }}$}

\begin{tabular}{|c|c|c|c|c|}
\hline \multirow[t]{5}{*}{ Spray Program \#12 } & $\underline{\text { Materials/Ing redients }}$ & $\begin{array}{l}\text { Amount } \\
\text { /Acre }\end{array}$ & $\underline{\text { Cost/Acre }}$ & $\begin{array}{c}\text { Your } \\
\text { Cost/Acre } \\
\end{array}$ \\
\hline & Kelthane MF & $6 \mathrm{pts}$ & $\$ 28.08$ & \\
\hline & Spray Buffer & $1 \mathrm{pt}$ & 2.03 & \\
\hline & $\begin{array}{l}\text { Ground Application } \\
\text { (PTO driven airblast) }\end{array}$ & 150 gals & $\underline{25.21}$ & \\
\hline & Total per Application & & $\$ 55.32$ & \\
\hline
\end{tabular}


Table 1-A.--Spray program s (cont'd.)

FALL SPRAY (cont'd.)

\begin{tabular}{|c|c|c|c|c|}
\hline \multirow[t]{4}{*}{ Spray Program \#13 } & $\underline{\text { Materials/Ing redients }}$ & $\begin{array}{l}\text { Amount } \\
\text { /Acre } \\
\end{array}$ & $\underline{\text { Cost/Acre }}$ & $\begin{array}{c}\text { Your } \\
\text { Cost/Acre } \\
\end{array}$ \\
\hline & Vendex 50WP & $2 \mathrm{lbs}$ & $\$ 32.72$ & \\
\hline & $\begin{array}{l}\text { Ground Application } \\
\text { (engine driven airblast) }\end{array}$ & 150 gals & $\underline{26.83}$ & \\
\hline & Total per Application & & $\$ \underline{\underline{59.55}}$ & \\
\hline \multirow[t]{4}{*}{ Spray Program \#14 } & $\underline{\text { Materials/Ing redients }}$ & $\begin{array}{l}\text { Amount } \\
\text { /Acre }\end{array}$ & $\underline{\text { Cost/Acre }}$ & $\begin{array}{c}\text { Your } \\
\text { Cost/Acre } \\
\end{array}$ \\
\hline & Thiolux (Sulfur) & $15 \mathrm{lbs}$ & $\$ 11.10$ & \\
\hline & $\begin{array}{l}\text { Ground Application } \\
\quad \text { (PTO driven airblast) }\end{array}$ & 150 gals & $\underline{25.21}$ & \\
\hline & Total per Application & & $\$ 36.31$ & \\
\hline
\end{tabular}

Table 2-A.--Herbicide

\begin{tabular}{|c|c|c|c|c|}
\hline Herbicide Program \#1 & $\underline{\text { Materials }}$ & $\begin{array}{c}\text { Amount/ } \\
\text { Treated Acre }\end{array}$ & $\begin{array}{c}\text { Cost/ } \\
\text { Grove Acre }^{\mathrm{a}}\end{array}$ & $\begin{array}{r}\text { Your Cost/ } \\
\text { Grove Acre } \\
\end{array}$ \\
\hline \multirow{5}{*}{ (Strip/band) } & Solicam $80 \mathrm{DF}$ & $3 \mathrm{lbs}$ & $\$ 24.87$ & \\
\hline & Karmex WP & $4 \mathrm{lbs}$ & 8.12 & \\
\hline & Roundup Ultra Max & 2 qts & 12.10 & \\
\hline & $\begin{array}{l}\text { Ground Application } \\
\text { (1 time) }\end{array}$ & & $\underline{13.81}$ & \\
\hline & Total for 1 Application & & $\$ \underline{\underline{58.90}}$ & \\
\hline \multirow{6}{*}{$\begin{array}{l}\text { Herbicide Program \#2 } \\
\text { (Strip/band) }\end{array}$} & $\underline{\text { Materials }}$ & $\begin{array}{c}\text { Amount/ } \\
\text { Treated Acre } \\
\end{array}$ & $\begin{array}{c}\text { Cost/ } \\
\text { Grove Acre }^{\mathrm{a}}\end{array}$ & $\begin{array}{r}\text { Your Cost/ } \\
\text { Grove Acre }\end{array}$ \\
\hline & Mandate & $2 \mathrm{pts}$ & $\$ 24.83$ & \\
\hline & Direx 4L & 3 qts & 7.14 & \\
\hline & Roundup Ultra Max & 2 qts & 12.10 & \\
\hline & $\begin{array}{l}\text { Ground Application } \\
\text { (1 time) }\end{array}$ & & $\underline{13.81}$ & \\
\hline & Total for 1 Application & & $\$ \underline{\underline{57.88}}$ & \\
\hline \multirow{5}{*}{$\begin{array}{l}\text { Herbicide Program \#3 } \\
\text { (Strip/band) }\end{array}$} & $\underline{\text { Materials }}$ & $\begin{array}{c}\text { Amount/ } \\
\text { Treated Acre }\end{array}$ & $\begin{array}{c}\text { Cost } / \\
\text { Grove Acre }^{\mathrm{a}}\end{array}$ & $\begin{array}{r}\text { Your Cost/ } \\
\text { Grove Acre } \\
\end{array}$ \\
\hline & Karmex WP & $4 \mathrm{lbs}$ & $\$ 8.12$ & \\
\hline & Roundup Ultra Max & 2 qts & 12.10 & \\
\hline & $\begin{array}{l}\text { Ground Application } \\
\text { (1 time) }\end{array}$ & & $\underline{13.81}$ & \\
\hline & Total for 1 Application & & $\$ 34.03$ & \\
\hline
\end{tabular}


Table 2-A.--Herbicide (cont'd.)

\begin{tabular}{|c|c|c|c|c|}
\hline Herbicide Program \#4 & $\underline{\text { Materials }}$ & $\begin{array}{c}\text { Amount/ } \\
\text { Treated Acre }\end{array}$ & $\begin{array}{c}\text { Cost/ } \\
\text { Grove Acre }^{\mathrm{a}}\end{array}$ & $\begin{array}{r}\text { Your Cost/ } \\
\text { Grove Acre } \\
\end{array}$ \\
\hline \multirow[t]{4}{*}{ (Strip/band) } & Roundup Ultra Max & $2 \mathrm{qts}$ & $\$ 12.10$ & \\
\hline & Ammon ium Sulfate & $17 \mathrm{lbs}$ & 1.32 & \\
\hline & $\begin{array}{l}\text { Ground Application } \\
\text { (1 time) }\end{array}$ & & $\underline{13.81}$ & \\
\hline & Total for 1 Application & & $\$ 27.83$ & \\
\hline \multirow{4}{*}{$\begin{array}{l}\text { Herbicide Program \#5 } \\
\text { (Strip/band) }\end{array}$} & $\underline{\text { Materials }}$ & $\begin{array}{c}\text { Amount/ } \\
\text { Treated Acre }\end{array}$ & $\begin{array}{l}\text { Cost/ } \\
\text { Grove Acre }^{\text {a }}\end{array}$ & $\begin{array}{r}\text { Your Cost/ } \\
\text { Grove Acre } \\
\end{array}$ \\
\hline & $\begin{array}{l}\text { Roundup Ultra Max } \\
\text { Princep (Caliber 90) }\end{array}$ & $\begin{array}{l}2 \mathrm{qts} \\
4 \mathrm{lbs}\end{array}$ & $\begin{array}{r}\$ 12.10 \\
6.60\end{array}$ & \\
\hline & $\begin{array}{l}\text { Ground Application } \\
\quad(1 \text { time })\end{array}$ & & $\underline{13.81}$ & \\
\hline & Total for 1 Application & & $\$ 32.51$ & \\
\hline \multirow{6}{*}{$\begin{array}{l}\text { Herbicide Program \#6 } \\
\text { (Strip/band) }\end{array}$} & Materials & $\begin{array}{c}\text { Amount/ } \\
\text { Treated Acre }\end{array}$ & $\begin{array}{c}\text { Cost/ } \\
\text { Grove Acre }^{\mathrm{a}}\end{array}$ & $\begin{array}{r}\text { Your Cost/ } \\
\text { Grove Acre } \\
\end{array}$ \\
\hline & Direx 4L & 3 qts & $\$ 7.14$ & \\
\hline & Solicam & $3 \mathrm{lbs}$ & 24.87 & \\
\hline & Roundup Ultra Max & 2 qts & 12.10 & \\
\hline & $\begin{array}{l}\text { Ground Application } \\
\text { (1 time) }\end{array}$ & & $\underline{13.81}$ & \\
\hline & Total for 1 Application & & $\$ \underline{\underline{57.92}}$ & \\
\hline \multirow{4}{*}{$\begin{array}{l}\text { Herbicide Program \#7 } \\
\text { (Spot herbicide for } \\
\text { grass/brush regrowth } \\
\text { under trees.) }\end{array}$} & $\underline{\text { Materials }}$ & $\begin{array}{c}\text { Amount/ } \\
\text { Treated Acre }\end{array}$ & $\begin{array}{c}\text { Cost } / \\
\text { Grove Acre }^{\mathrm{a}}\end{array}$ & $\begin{array}{r}\text { Your Cost/ } \\
\text { Grove Acre } \\
\end{array}$ \\
\hline & Roundup Ultra Max & 2 qts & $\$ 12.10$ & \\
\hline & $\begin{array}{l}\text { Ground Application } \\
\quad(1 \text { time })\end{array}$ & 15 gals & 7.13 & \\
\hline & Total for 1 Application & & $\$ 19.23$ & \\
\hline
\end{tabular}

${ }^{a}$ With respect to herbicide materials, Amount P er Grove Acre does not equal Amount Per Treated Acre shown on the label. Only a strip or band is being treated. In this report, it is assumed that only one-half of a grove surface is being treated. 
Table 3-A.--Dry fertilizer

\begin{tabular}{|c|c|c|c|c|}
\hline Program \#1 & $\begin{array}{l}\text { Analysis/Material } \\
\text { Applied }\end{array}$ & $\begin{array}{l}\text { Amount } \\
\text { /Acre } \\
\end{array}$ & $\underline{\text { Cost/Acre }}$ & $\begin{array}{c}\text { Your } \\
\text { Cost/Acre }\end{array}$ \\
\hline \multirow{3}{*}{ (162 lbs N/Acre) } & $12-2-12-2.4 \mathrm{MgO}$ & $1350 \mathrm{lbs}$ & \$ 109.35 & \\
\hline & Application & 3 times & $\underline{26.13}$ & \\
\hline & Total for 3 Applications & & $\$ \underline{\underline{135.48}}$ & \\
\hline \multirow{4}{*}{$\begin{array}{l}\text { Program \#2 } \\
\qquad(180 \mathrm{lbs} \text { N/Acre })\end{array}$} & $\begin{array}{l}\text { Analysis/Material } \\
\text { Applied }\end{array}$ & $\begin{array}{l}\text { Amount } \\
\text { /Acre } \\
\end{array}$ & $\underline{\text { Cost/Acre }}$ & $\begin{array}{c}\text { Your } \\
\text { Cost/Acre }\end{array}$ \\
\hline & $16-0-16-4 \mathrm{MgO}$ & $1125 \mathrm{lbs}$ & $\$ 108.00$ & \\
\hline & Application & 3 times & 26.13 & \\
\hline & Total for 3 Applications & & $\$ \underline{\underline{134.13}}$ & \\
\hline \multirow{4}{*}{$\begin{array}{l}\text { Program \#3 } \\
\text { (204 lbs N/Acre) }\end{array}$} & $\begin{array}{l}\text { Analysis/Material } \\
\text { Applied }\end{array}$ & $\begin{array}{l}\text { Amount } \\
\text { /Acre } \\
\end{array}$ & $\underline{\text { Cost/Acre }}$ & $\begin{array}{c}\text { Your } \\
\text { Cost/Acre } \\
\end{array}$ \\
\hline & $16-0-16-4 \mathrm{MgO}$ & $1275 \mathrm{lbs}$ & $\$ 122.40$ & \\
\hline & Application & 3 times & $\underline{26.13}$ & \\
\hline & Total for 3 Applications & & $\$ 148.53$ & \\
\hline \multirow{4}{*}{$\begin{array}{l}\text { Program \#4 } \\
\text { (225 lbs N/Acre) }\end{array}$} & $\begin{array}{l}\text { Analysis/Material } \\
\text { Applied }\end{array}$ & $\begin{array}{l}\text { Amount } \\
\text { /Acre } \\
\end{array}$ & $\underline{\text { Cost/Acre }}$ & $\begin{array}{c}\text { Your } \\
\text { Cost/Acre }\end{array}$ \\
\hline & $15-2-15-2.4 \mathrm{MgO}$ & $1500 \mathrm{lbs}$ & $\$ 136.50$ & \\
\hline & Application & 3 times & $\underline{26.13}$ & \\
\hline & Total for 3 Applications & & $\$ \underline{\underline{162.63}}$ & \\
\hline
\end{tabular}


Table 4-A.--Liquid fertilizer (Double boom application)

\begin{tabular}{|c|c|c|c|c|}
\hline \multirow{4}{*}{$\begin{array}{l}\text { Program \#1 } \\
(180 \mathrm{lbs} \text { N/Acre })\end{array}$} & $\begin{array}{l}\text { Analysis/Material } \\
\text { Applied } \\
\end{array}$ & $\begin{array}{l}\text { Amount } \\
\text { /Acre }\end{array}$ & Cost/Acre & $\begin{array}{c}\text { Your } \\
\text { Cost/Acre } \\
\end{array}$ \\
\hline & $10-0-10$ & $1800 \mathrm{lbs}$ & $\$ 111.60$ & \\
\hline & Double Boom Application & 3 times & $\underline{43.50}$ & \\
\hline & Total for 3 Applications & & $\$ \underline{\underline{155.10}}$ & \\
\hline \multirow{4}{*}{$\begin{array}{l}\text { Program \#2 } \\
\text { (180 lbs N/Acre) }\end{array}$} & $\begin{array}{l}\text { Analysis/Material } \\
\text { Applied }\end{array}$ & $\begin{array}{l}\text { Amount } \\
\text { /Acre }\end{array}$ & $\underline{\text { Cost/Acre }}$ & $\begin{array}{c}\text { Your } \\
\text { Cost/Acre } \\
\end{array}$ \\
\hline & $10-2-10$ & $1800 \mathrm{lbs}$ & $\$ 117.00$ & \\
\hline & Double Boom Application & 3 times & $\underline{43.50}$ & \\
\hline & Total for 3 Applications & & $\$ \underline{\underline{160.50}}$ & \\
\hline \multirow{7}{*}{$\begin{array}{l}\text { Program \#3 } \\
\text { (180 lbs N/Acre) }\end{array}$} & $\begin{array}{l}\text { Analysis/Material } \\
\text { Applied }\end{array}$ & $\begin{array}{l}\text { Amount } \\
\text { /Acre } \\
\end{array}$ & $\underline{\text { Cost/Acre }}$ & $\begin{array}{c}\text { Your } \\
\text { Cost/Acre } \\
\end{array}$ \\
\hline & $10-0-10$ & $1800 \mathrm{lbs}$ & $\$ 111.60$ & \\
\hline & Solicam $80 \mathrm{DF}$ & 3 lbs* & 24.87 & \\
\hline & Karmex WP & 4 lbs* & 8.12 & \\
\hline & Double Boom Application & 3 times & $\underline{43.50}$ & \\
\hline & Total for 3 Applications & & $\$ \underline{\underline{188.09}}$ & \\
\hline & \multicolumn{4}{|c|}{ *Treated acre (one herbicide application) } \\
\hline
\end{tabular}

Table 5-A.--Nematicides

\begin{tabular}{|c|c|c|c|c|}
\hline \multirow[t]{4}{*}{ Program \#1 } & $\begin{array}{l}\text { Analysis/Material } \\
\text { Applied }\end{array}$ & $\begin{array}{l}\text { Amount } \\
\text { /Acre }\end{array}$ & $\underline{\text { Cost/Acre }}$ & $\begin{array}{c}\text { Your } \\
\text { Cost/Acre } \\
\end{array}$ \\
\hline & Temik 15G & $33 \mathrm{lbs}$ & $\$ 113.85$ & \\
\hline & Application & & 14.26 & \\
\hline & Total per Application & & $\$ \underline{\underline{128.11}}$ & \\
\hline \multirow[t]{4}{*}{ Program \#2 } & $\begin{array}{l}\text { Analysis/Material } \\
\text { Applied }\end{array}$ & $\begin{array}{l}\text { Amount } \\
\text { /Acre }\end{array}$ & $\underline{\text { Cost/Acre }}$ & $\begin{array}{c}\text { Your } \\
\text { Cost/Acre } \\
\end{array}$ \\
\hline & Temik 15G & $17 \mathrm{lbs}$ & $\$ 58.65$ & \\
\hline & Application & & $\underline{14.26}$ & \\
\hline & Total per Application & & $\$ 72.91$ & \\
\hline
\end{tabular}


Table 6-A.--Soil amendment

\begin{tabular}{|c|c|c|c|c|}
\hline Program \#1 & $\begin{array}{l}\text { Analysis/Material } \\
\text { Applied } \\
\end{array}$ & $\begin{array}{l}\text { Amount } \\
\text { /Acre } \\
\end{array}$ & $\underline{\text { Cost/Acre }}$ & $\begin{array}{c}\text { Your } \\
\text { Cost/Acre } \\
\end{array}$ \\
\hline \multirow[t]{4}{*}{ (Every 3 years) } & Dolomite (Delivered) & 1 ton & $\$ 30.14$ & \\
\hline & Application & 1 time & 8.80 & \\
\hline & Total for 1 Application & & $\$ \underline{\underline{38.94}}$ & \\
\hline & (Average $1 / 3$ Ton Applied/Yr) & & $\$ \underline{\underline{12.98}}$ & \\
\hline \multirow{5}{*}{$\begin{array}{l}\text { Program } \# 2 \\
\text { (Every } 4 \text { years) }\end{array}$} & $\begin{array}{l}\text { Analysis/Material } \\
\text { Applied } \\
\end{array}$ & $\begin{array}{l}\text { Amount } \\
\text { /Acre }\end{array}$ & Cost/Acre & $\begin{array}{c}\text { Your } \\
\text { Cost/Acre } \\
\end{array}$ \\
\hline & Dolomite (Delivered) & 1 ton & $\$ 30.14$ & \\
\hline & Application & 1 time & 8.80 & \\
\hline & Total for 1 Application & & $\$ \underline{\underline{38.94}}$ & \\
\hline & (Average $1 / 4$ Ton Applied/Yr) & & $\$ 9.74$ & \\
\hline
\end{tabular}

Table 7-A.--Irrigation--annual cost per ac re

\section{PERMANENT OVERHEAD}

\begin{tabular}{|c|c|c|c|c|}
\hline & $\underline{\text { Program \#1 }}$ & $\begin{array}{c}\text { Your } \\
\text { Cost/Acre } \\
\end{array}$ & $\underline{\text { Program \#2 }}$ & $\begin{array}{c}\text { Your } \\
\text { Cost/Acre }\end{array}$ \\
\hline Operating & $\begin{array}{c}\text { (Electric) } \\
\$ 103.60\end{array}$ & & $\begin{array}{c}(\text { Diesel) } \\
\$ 84.15\end{array}$ & \\
\hline Maintenance of System & 41.83 & & 43.88 & \\
\hline Total Cash Expenses & $\$ 145.43$ & & $\$ 128.03$ & \\
\hline Fixed Depreciation Expense & 55.73 & & 59.54 & \\
\hline Total Cash and Fixed Expenses & $\$ 201.16$ & & $\$ 187.57$ & \\
\hline \multicolumn{5}{|l|}{ MICROSPRINKLER } \\
\hline & $\underline{\text { Program \#3 }}$ & $\begin{array}{c}\text { Your } \\
\text { Cost/Acre } \\
\end{array}$ & $\underline{\text { Program \#4 }}$ & $\begin{array}{c}\text { Your } \\
\text { Cost/Acre }\end{array}$ \\
\hline Operating & $\begin{array}{c}\text { (Electric) } \\
\$ 49.87\end{array}$ & & $\begin{array}{l}\text { (Diesel) } \\
\$ 41.98\end{array}$ & \\
\hline Maintenance of System & 45.75 & & 46.76 & \\
\hline Total Cash Expenses & $\$ 95.62$ & & $\$ 88.74$ & \\
\hline Fixed Depreciation Expense & 52.94 & 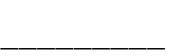 & 56.56 & \\
\hline Total Cash and Fixed Expenses & $\$ 148.56$ & & $\$ 145.30$ & \\
\hline
\end{tabular}


Table 8-A.-- A listing of 2003 custom rates reported by thirty Ridge citrus ca retakers

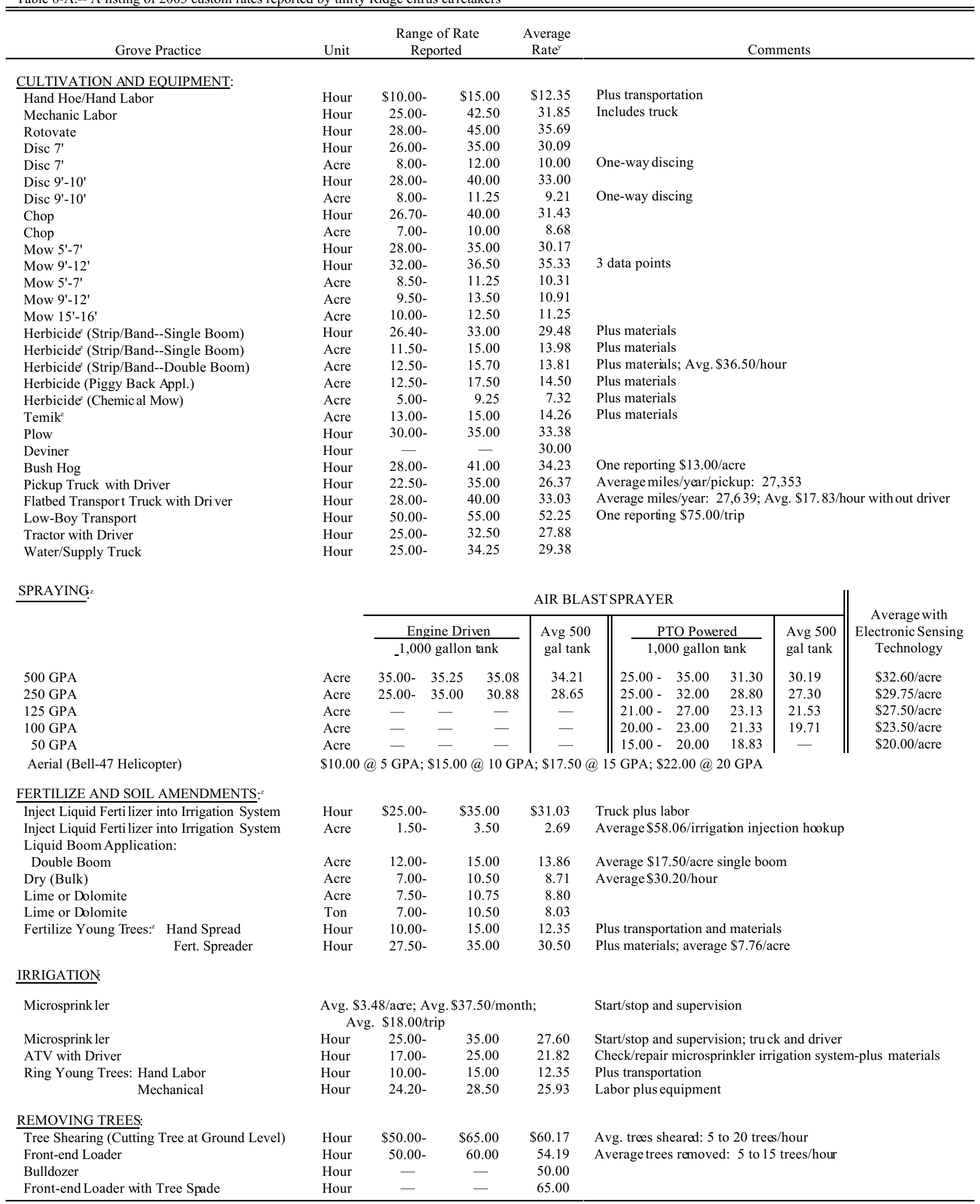


Table 8-A.-- A listing of 2003 custom rates reported by thirty Ridge citrus caretakers (cont'd.)

\begin{tabular}{|c|c|c|c|c|c|}
\hline Grove Practice & Unit & \multicolumn{2}{|c|}{$\begin{array}{l}\text { Range of Rate } \\
\text { Reported }\end{array}$} & $\begin{array}{l}\text { Average } \\
\text { Rate }^{\mathrm{y}}\end{array}$ & Comments \\
\hline \multicolumn{6}{|l|}{ PRUNING: } \\
\hline 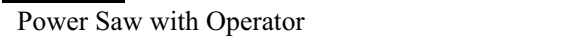 & Hour & $\$ 17.00-$ & $\$ 25.00$ & $\$ 20.50$ & Plus transportation \\
\hline Limb Lifter/Tree Skirt Trimmer (Double Sided) & Hour & - & - & 200.00 & Cover $9-18$ acres one pass \\
\hline \multicolumn{6}{|l|}{ Hedging: } \\
\hline Single Side(Tractor Mounted) & Hour & $75.00-$ & 80.00 & 76.67 & Cover $2-5$ acres/hour \\
\hline Double Side(Tractor Pulled) & Hour & $80.00-$ & 100.00 & 86.88 & Cover 3-5 acres/hour \\
\hline Double Side(Tractor Mounted) & Hour & $200.00-$ & 220.00 & 210.00 & \\
\hline Double Side (Self Propelled) & Hour & $325.00-$ & 360.00 & 341.67 & Cover $10-25$ ac res/hour depen ding on wood size \\
\hline Double Side (Self Propelled) & Hour & $250.00-$ & 300.00 & 287.50 & Cover 4-12 acres/hour depend ing on wood size \\
\hline \multicolumn{6}{|l|}{ Topping: } \\
\hline Tractor Mounted & Hour & $195.00-$ & 200.00 & 196.67 & \\
\hline Tractor Pulled & Hour & - & - & 100.00 & Cover $1-3$ acres/hour \\
\hline Self Propelled & Hour & $365.00-$ & 380.00 & 372.50 & Cover 5-10 acres/hr (Roof Top); 5-20 acres/hr (Flat Top) \\
\hline Double Boom (Self Propelled) & Hour & - & - & 550.00 & Cover 2-12 acres/hr (Roof Top); $15-30$ acres/hr (Flat Top) \\
\hline \multicolumn{6}{|l|}{ Removing Brush: } \\
\hline Haul Brush out of Grove & Hour & $35.00-$ & 40.50 & 37.10 & Tractor-trailer/truck, driver plus 1 person; plus 2 people \\
\hline Front-end Loader (Push Brush) & Hour & 46.40- & 60.00 & 53.72 & $2-10$ acres/hour \\
\hline Chop/Mow Brush & Hour & $27.90-$ & 37.50 & 33.42 & 3-6 acres/hour; Averaged \$11.31/acre \\
\hline \multicolumn{6}{|l|}{ COLD PROTECTION: } \\
\hline 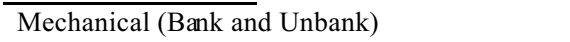 & Hour & $\$-$ & $\$-$ & $\$ 23.43$ & \\
\hline Install Wraps & Each & 0.24 & 0.50 & 0.34 & \\
\hline \multicolumn{6}{|l|}{ OTHER CUSTOM RATES: } \\
\hline $\begin{array}{l}\text { Plant Resets } \\
\end{array}$ & Per Tree & $\$ 1.75-$ & $\$ 3.00$ & $\$ 2.38$ & Stake, plant and first watering \\
\hline Solid Set Planting & Per Tree & $1.00-$ & 1.75 & 1.29 & Stake, plant and first watering \\
\hline Travel/Setup Charge & Hour & - & - & 27.00 & Average for those reporting; One reporting $\$ 100.00$ \\
\hline \multicolumn{6}{|l|}{ Grove Management Charge/Month: } \\
\hline Supervising Grove Care Operations & Acre & $1.50-$ & 5.50 & 2.88 & In addition to caretaking charges; $6 \%$ equipment labor \\
\hline Handling Fruit Marketing & Box & $0.10-$ & 0.25 & 0.15 & lcharge; $\$ 50 /$ acre annually \\
\hline Supervising/H andling Chem icals/Fertilizer & \multicolumn{5}{|c|}{$10 \%$ to $25 \%$ of materials cost } \\
\hline \multicolumn{6}{|l|}{$\begin{array}{l}\text { Charge for personnel to oversee harvesting } \\
\text { operations and coordinate harvest in different } \\
\text { blocks/groves and keeping of harvesting } \\
\text { labor compliance record. }\end{array}$} \\
\hline Consulting & \multicolumn{5}{|c|}{$\begin{array}{l}\text { Cultural Management/Horticultural Evaluation - } \$ 50 / \mathrm{hr} \text { to } \$ 100 / \mathrm{hr} \\
\text { Financial Analysis Prospectus - } \$ 150 / \mathrm{hr}\end{array}$} \\
\hline \multicolumn{6}{|l|}{ Total Reported Acreage Provided Grove } \\
\hline Service to: & Acre & $350-$ & 14,415 & 2,639 & Total acres reporting: 62,709 \\
\hline
\end{tabular}

\footnotetext{
${ }^{2}$ Plus materials. Caretakers reporting rates include labor, tractor and sprayer; supply truck included by most caretakers.

${ }^{y}$ Calculated by dividing the total numb er of caretakers reporting a grove practice rate into the sum reported. Unless otherwise stated, labor in cluded with all charges.

${ }^{\mathrm{x}}$ Low acres is for 2 years regrowth hedging; high acres is for annual maintenance hedging.
}

Source: Ronald P. Muraro, Extension Farm Management Economist, Lake Alfred CREC, June 2003. 
Table 9-A.--2003 summary of average chemical price estimates

\begin{tabular}{|c|c|c|c|c|}
\hline Item & & Unit & $\begin{array}{l}\text { Average } \\
\text { Price }\end{array}$ & $\begin{array}{c}\text { Your Price } \\
(2003)\end{array}$ \\
\hline Fungicides: & $\begin{array}{l}\text { Abound } \\
\text { Aliette 80WP } \\
\text { Basic Copper Sulfate (53\%) } \\
\text { Copper (50\%) (Kocide 101) } \\
\text { Carbamate 76WP } \\
\text { Enable 2F } \\
\text { Headline } \\
\text { Nu-Cop 50DF } \\
\text { Oil - 435 or 455 } \\
\text { Ridomil Gold Granular } \\
\text { Ridomil Gold EC }\end{array}$ & $\begin{array}{l}\text { gal. } \\
\text { lb. } \\
\text { lb. } \\
\text { lb. } \\
\text { lb. } \\
40 \mathrm{oz} . \\
\text { gal. } \\
\text { lb. } \\
\text { gal. } \\
\text { lb. } \\
\text { gal. }\end{array}$ & $\begin{array}{r}235.00 \\
9.96 \\
1.27 \\
1.96 \\
3.13 \\
56.40 \\
220.00 \\
1.80 \\
2.25 \\
5.35 \\
604.15\end{array}$ & \\
\hline \multicolumn{5}{|c|}{$\underline{\text { Insecticides/Nematicides: }}$} \\
\hline & $\begin{array}{l}\text { Admire 2F } \\
\text { Agri-Mek }(0.15 \mathrm{EC}) \\
\text { Bacillus thuringienses } \\
\text { Comite } 6.55 \mathrm{EC} \\
\text { Danitol } \\
\text { Ethion } \\
\text { Guthion 50WP } \\
\text { Kelthane MF } \\
\text { Lorsban 4EC } \\
\text { Lorsban 15G } \\
\text { Malathion 5 EC } \\
\text { Micromite 25WP } \\
\text { Microthiol 80DF } \\
\text { Nexter WP } \\
\text { Sevin 80S } \\
\text { Sevin XLR } \\
\text { Sulphur 6F } \\
\text { Temik 15G } \\
\text { Thiolux 80 DF } \\
\text { Vendex 50W }\end{array}$ & $\begin{array}{l}\text { gal. } \\
\text { gal. } \\
\text { gal. } \\
\text { gal. } \\
\text { gal. } \\
\text { gal. } \\
\text { lb. } \\
\text { gal. } \\
\text { gal. } \\
\text { lb. } \\
\text { gal. } \\
\text { lb. } \\
\text { lb. } \\
\text { lb. } \\
\text { lb. } \\
\text { gal. } \\
\text { gal. } \\
\text { lb. } \\
\text { lb. } \\
\text { lb. }\end{array}$ & $\begin{array}{r}522.50 \\
604.00 \\
9.92 \\
80.95 \\
141.67 \\
33.75 \\
9.78 \\
34.00 \\
41.25 \\
1.76 \\
20.09 \\
31.22 \\
0.67 \\
100.44 \\
4.57 \\
25.93 \\
2.65 \\
3.14 \\
0.67 \\
14.88\end{array}$ & L \\
\hline
\end{tabular}


Table 9-A.--2003 summary of average chemical price estimates (cont'd.)

\begin{tabular}{|c|c|c|c|c|}
\hline \multicolumn{2}{|l|}{ Item } & Unit & $\begin{array}{c}\text { Average } \\
\text { Price }\end{array}$ & $\begin{array}{c}\text { Your Price } \\
(2003) \\
\end{array}$ \\
\hline \multirow[t]{19}{*}{$\underline{\text { Herbicides: }}$} & Direx 4L & gal. & 17.24 & \\
\hline & Direx $80 \mathrm{DF}$ & lb. & 3.49 & \\
\hline & Fusilade DX & gal. & 123.57 & \\
\hline & Gramoxone (Paraquat) & gal. & 33.65 & \\
\hline & Hyvar X & lb. & 18.19 & \\
\hline & Karmex & lb. & 3.69 & \\
\hline & Krovar I & lb. & 10.68 & \\
\hline & Mandate 2E & gal. & 180.63 & \\
\hline & Poast Plus 1.0 EC & gal. & 53.97 & \\
\hline & Princep (Caliber 90) & lb. & 3.00 & \\
\hline & Princep 4L & gal. & 13.71 & \\
\hline & Rodeo (30 gal drum) & gal. & 72.69 & \\
\hline & Roundup (30 gal drum) & gal. & 27.75 & \\
\hline & Roundup Ultra Max & gal. & 44.00 & \\
\hline & Simazine $90 \mathrm{DF}$ & lb. & 2.72 & \\
\hline & Simazine 4L & gal. & 13.00 & \\
\hline & Solicam 80 DF & lb. & 15.08 & \\
\hline & Surflan AS & gal. & 70.73 & \\
\hline & Touchdown & gal. & 35.67 & \\
\hline \multicolumn{5}{|c|}{ Growth Regulators: } \\
\hline & Citrus Fix & gal. & 284.48 & \\
\hline & Pro-Gibb 3.91\% & 32 oz. bottle & 37.56 & \\
\hline \multicolumn{5}{|c|}{ Other Spray Materials: } \\
\hline & Borates $(15 \%)$ & lb. & 0.74 & \\
\hline & Manganese $(32 \%)$ & lb. & 0.31 & \\
\hline & Zinc $(78 \%)$ & lb. & 0.79 & \\
\hline & Nutritional Spray Mix: & & & \\
\hline & Dyna Gold MZF & gal. & 6.41 & \\
\hline
\end{tabular}

SOURCE: Ronald P. Muraro, Extension Farm Management Economist, University of Florida, IFAS, CREC, Lake Alfred, Florida, August 2003. 
Table 10-A.--2003 summary of average fertilizer price estimates

\begin{tabular}{|c|c|c|c|}
\hline Item & Unit & $\begin{array}{c}\text { Average } \\
\text { Price } \\
\end{array}$ & $\begin{array}{c}\text { Your Price } \\
(2003) \\
\end{array}$ \\
\hline \multicolumn{4}{|c|}{ FERTILIZER (FOB Price @ Plant) } \\
\hline & & $\$$ & \\
\hline \multicolumn{4}{|l|}{ Dry Mix (Bulk) } \\
\hline $17-0-17-3_{\mathrm{Mg}}$ & ton & 177.44 & \\
\hline $17-4-17-2.4_{\mathrm{Mg}}$ & ton & 180.46 & \\
\hline $16-0-16$ & ton & 166.08 & \\
\hline $16-0-16-4_{\mathrm{Mg}}$ & ton & 174.36 & \\
\hline $16-2-16-3_{\mathrm{Mg}}$ & ton & 175.82 & \\
\hline $15-2-15-2.4_{\mathrm{Mg}}$ & ton & 165.26 & \\
\hline $12-2-12-2.4_{\mathrm{Mg}}$ & ton & 147.78 & \\
\hline $8-8-8 \mathrm{w} / \mathrm{minors} *$ & ton & 145.30 & \\
\hline $8-4-8 \mathrm{w} / \mathrm{minors} *$ & ton & 128.44 & \\
\hline $8-2-8 \mathrm{w} / \mathrm{minors} *$ & ton & 126.26 & \\
\hline $6-6-6 \mathrm{w} / \mathrm{minors} *$ & ton & 127.03 & \\
\hline \multicolumn{4}{|l|}{$\underline{\text { Liquid Mix (Bulk) }}$} \\
\hline $8-2-8$ & ton & 112.48 & \\
\hline $8-4-8$ & ton & 107.08 & \\
\hline $9-3-9$ & ton & 109.54 & \\
\hline $9-4-9$ & ton & 124.19 & \\
\hline $10-0-10$ & ton & 111.81 & \\
\hline $10-2-10$ & ton & 118.71 & \\
\hline $12-0-6$ & ton & 110.49 & \\
\hline $12-3-6$ & ton & 108.41 & \\
\hline
\end{tabular}

*With organic nitrogen, the price averaged $25 \%$ higher. 
Table 10-A.--2003 summary of average fertilizer price estimates (cont'd.)

\begin{tabular}{|c|c|c|c|}
\hline Item & Unit & $\begin{array}{c}\text { Average } \\
\text { Price }\end{array}$ & $\begin{array}{c}\text { Your Price } \\
(2003) \\
\end{array}$ \\
\hline \multicolumn{4}{|l|}{ Other Fertilizer Materials (Bulk) } \\
\hline Ammonium Nitrate (21\% N Liquid) & ton & 124.75 & \\
\hline Ammonium Nitrate (33.5\% N Dry) & ton & 187.38 & \\
\hline Ammonium Sulfate $(21 \% \mathrm{~N})$ & ton & 101.67 & \\
\hline Calcium Nitrate $(19 \% \mathrm{Ca}, 15.5 \% \mathrm{~N})$ & ton & 207.69 & \\
\hline Dolomite (at mine--49\% $\mathrm{CaCO}_{3}, 36 \% \mathrm{MgCO}_{3}$ ) & ton & 16.49 & \\
\hline Muriate of Potash $\left(60 \% \mathrm{~K}_{2} \mathrm{O}\right)$ & ton & 162.02 & \\
\hline Potassium Nitrate $\left(14 \% \mathrm{~N} ; 46 \% \mathrm{~K}_{2} \mathrm{O}\right)$ & ton & 373.26 & \\
\hline Sul-Po-Mag (SPM--21.9\% $\left.\mathrm{K}_{2} \mathrm{O}\right)$ & ton & 162.50 & \\
\hline Super Phosphate $\left(20 \% \mathrm{P}_{2} \mathrm{O}_{5}\right)$ & ton & 143.31 & \\
\hline Triple Superphosphate $\left(48 \% \mathrm{P}_{2} \mathrm{O}_{5}\right)$ & ton & 172.94 & \\
\hline Urea & ton & 373.26 & \\
\hline Average Delivery Cost & ton & 12.00 & \\
\hline \multicolumn{4}{|l|}{$\underline{\text { Foliar Macronutrients }}$} \\
\hline N-Sure $28-0-0(72 \% \mathrm{SRN})^{* *}$ & gal. & 6.39 & \\
\hline Phos Might 0-22-20 & gal. & 21.87 & \\
\hline Nutriphite $0-28-26$ & gal. & 26.67 & \\
\hline MKP (0-52-34) (Mono-Potassium Phosphate) & lb. & 0.65 & \\
\hline DKP (0-18-20) (Di-Potassium Phosphate) & gal. & 2.50 & \\
\hline
\end{tabular}

**SRN, Slow Release Nitrogen

SOURCE: Ronald P. Muraro, Extension Farm Management Economist, University of Florida, IFAS, CREC, Lake Alfred, Florida, August 2003. 
Table 11-A.--Cost for establishing, planting and maintaining a citrus grove through four years of age, North Florida area

\begin{tabular}{|c|c|c|c|c|}
\hline & & \multicolumn{3}{|c|}{ Cost Per Acre } \\
\hline & & \multicolumn{2}{|c|}{ Range } & Average \\
\hline & & \multicolumn{2}{|l|}{$\$$} & $\$$ \\
\hline Land $\operatorname{Cos} t^{1}$ & & \multicolumn{2}{|c|}{$2,500-4,500$} & 3,450 \\
\hline Land Preparation (clearing, disking, leveling) & & \multicolumn{2}{|c|}{$275-700^{2}$} & 350 \\
\hline Soil Amendments: Dolomite 1 ton & & & & 35 \\
\hline Super Phosphate, 400 lbs. & & \multirow{2}{*}{\multicolumn{2}{|c|}{$9-\quad 16$}} & 30 \\
\hline Cover Crop & & & & 12 \\
\hline \multirow{2}{*}{\multicolumn{2}{|c|}{ Irrigation System: Microsprinkler -- with Well ${ }^{3}$}} & \multicolumn{2}{|c|}{$850-1,650$} & 1,350 \\
\hline & & \multicolumn{2}{|c|}{$525-1,250$} & 975 \\
\hline \multirow[t]{2}{*}{ Water Permits, Environmental Studies, and Engineering: } & Cost & \multicolumn{2}{|c|}{$35-80$} & 50 \\
\hline & Time in Months & \multirow{3}{*}{\multicolumn{2}{|c|}{$\begin{array}{rc}3- & 8 \\
90 \%- & 97 \% \\
3 \%- & 10 \%\end{array}$}} & 6 \\
\hline \multirow{5}{*}{ Percent Land Utilization: Planted to Cit } & & & & $95 \%$ \\
\hline & & & & $5 \%$ \\
\hline & & \multicolumn{3}{|c|}{ North Florida } \\
\hline & & \multicolumn{2}{|c|}{ Year } & \\
\hline & 1 & 2 & 3 & 4 \\
\hline \multicolumn{2}{|l|}{$\underline{\text { Solidset Planted Trees }}^{4}$} & \multicolumn{3}{|c|}{ Cost Per Tree --------- } \\
\hline Microsprinkler Irrigation and Ditch Maintenance & $\$ 0.37$ & $\$ 0.45$ & $\$ 0.55$ & $\$ 0.65$ \\
\hline Fertilize Tree & 0.25 & 0.40 & 0.55 & 0.57 \\
\hline Supplemental Fertilization thru Irrigation & 0.15 & 0.20 & 0.25 & 0.29 \\
\hline Spray & 0.42 & 0.55 & 0.65 & 0.75 \\
\hline Insulated Tree Wrap (annual maintenance) & 0.25 & 0.25 & 0.25 & 0.00 \\
\hline Sprouting (labor) & 0.20 & 0.20 & 0.00 & 0.00 \\
\hline Cultivation/Mowing & 0.40 & 0.40 & 0.40 & 0.40 \\
\hline Herbicide & 0.45 & 0.45 & 0.45 & 0.45 \\
\hline Ridomil/A liette & 0.35 & 0.35 & 0.00 & 0.00 \\
\hline Miscellaneous & 0.43 & 0.49 & 0.47 & 0.47 \\
\hline Total Cost Per Year & $\overline{\$ 3.27}$ & $\overline{\$ 3.74}$ & $\overline{\$ 3.57}$ & $\overline{\$ 3.58}$ \\
\hline$\underline{\text { Reset Trees (annual additional grove care costs) }}$ & $\$ 2.13$ & $\$ 2.47$ & $\$ 1.84$ & -- \\
\hline Cost of Planting Trees ${ }^{5}$ & & Solidset $=\$ 6.75$ & \multicolumn{2}{|c|}{ Reset $=\$ 8.05$} \\
\hline
\end{tabular}

${ }^{1}$ Land cost will vary from one county to another as well as from one parcel to another.

${ }^{2}$ Includes fumigation cost of $\$ 330$ per acre.

${ }^{3}$ Irrigation costs include distribution system, power unit and well (where indicated.) The higher cost ranges reported also include $\mathrm{d}$ a cost for fertigation equipment.

${ }^{4}$ The per tree costs shown are applicable for tree densities of 145 to 165 trees per acre. The per tree costs should be decreased for higher density plantings and increased for lower density plantings; e.g., at 200 trees per acre decrease costs by $15 \%$; at 115 trees per acre increase costs by $15 \%$.

${ }^{5}$ Tree cost $($ bare root) $=\$ 3.25$; stake, plant, and water tree $=\$ 1.25$ (solidset) and $\$ 2.55$ (resets); and insulated tree wrap

$=\$ 2.25$.

Source: Ronald P. Muraro, Farm Management Economist, CREC, Lake Alfred, FL, March 1990. 
Table 12-A.--Estimated cost of planting and maintaining a reset citrus tree through three years of age, December 2000

\begin{tabular}{|c|c|c|c|c|c|}
\hline & \multicolumn{5}{|c|}{ Number of Resets/Replacement Trees Per Acre } \\
\hline & $1-2$ & $3-5$ & $6-10$ & $11-25$ & $26+$ \\
\hline & \multicolumn{5}{|c|}{---------------- Cost Per Tree ---------------- } \\
\hline$\underline{\text { Year \#1: }}$ & $\$$ & $\$$ & $\$$ & $\$$ & $\$$ \\
\hline Tree Removal & 5.73 & 4.98 & 3.98 & 3.22 & 2.57 \\
\hline Tree Cost (Container Tree) & 4.50 & 4.50 & 4.35 & 4.25 & 4.25 \\
\hline Site Preparation ${ }^{\mathrm{a}}$ & 5.40 & 4.68 & 3.97 & 3.67 & 2.88 \\
\hline Plant Tree and First Watering & $\underline{3.28}$ & $\underline{2.40}$ & $\underline{2.08}$ & $\underline{1.74}$ & $\underline{1.31}$ \\
\hline Total Planting Cost & $\overline{13.18}$ & $\overline{11.58}$ & $\overline{10.40}$ & $\overline{9.66}$ & $\overline{8.44}$ \\
\hline \multicolumn{6}{|l|}{ Supplemental Fertilization -4 Times } \\
\hline \multicolumn{6}{|l|}{ Supplemental Spraying } \\
\hline (Application \& Materials) $^{\mathrm{b}}$ & 0.48 & 0.41 & 0.38 & 0.35 & 0.32 \\
\hline Spot Herbicide (Application \& Materials) & 0.21 & 0.18 & 0.16 & 0.14 & 0.13 \\
\hline Tree Wrap (Corrugated) & 1.00 & 1.00 & 1.00 & 1.00 & 1.00 \\
\hline Sprouting/Pruning & 0.41 & 0.41 & 0.38 & 0.38 & 0.34 \\
\hline Miscellaneous & 0.17 & 0.16 & 0.15 & 0.14 & 0.13 \\
\hline Supervision \& Overhead & $\underline{0.27}$ & $\underline{0.25}$ & $\underline{0.23}$ & $\underline{0.22}$ & $\underline{0.21}$ \\
\hline Total Tree Care Cost Year \#1 & 3.82 & 3.52 & 3.32 & 3.16 & 2.98 \\
\hline Total Cost Year \#1 & 22.73 & 20.08 & 17.70 & 16.04 & 13.99 \\
\hline \multicolumn{6}{|l|}{ Year \#2: } \\
\hline \multicolumn{6}{|l|}{ Supplemental Fertilization -3 Times } \\
\hline (Application \& Materials) & 1.72 & 1.53 & 1.34 & 1.13 & 1.03 \\
\hline \multicolumn{6}{|l|}{ Supplemental Spraying } \\
\hline (Application \& Materials) $^{\mathrm{b}}$ & 0.55 & 0.49 & 0.43 & 0.36 & 0.33 \\
\hline Spot Herbicide (Application \& Materials) & 0.20 & 0.18 & 0.16 & 0.14 & 0.13 \\
\hline Sprouting/Pruning & 0.49 & 0.49 & 0.41 & 0.41 & 0.37 \\
\hline Miscellaneous & 0.15 & 0.13 & 0.12 & 0.10 & 0.09 \\
\hline Supervision \& Overhead & $\underline{0.23}$ & $\underline{0.20}$ & $\underline{0.18}$ & $\underline{0.16}$ & $\underline{0.15}$ \\
\hline Total Cost Year \#2 & $\overline{3.34}$ & $\overline{3.02}$ & $\overline{2.64}$ & $\overline{2.30}$ & $\overline{2.10}$ \\
\hline \multicolumn{6}{|l|}{$\underline{\text { Year \#3: }}$} \\
\hline \multicolumn{6}{|l|}{ Supplemental Fertilization -3 Times } \\
\hline (Application \& Materials) & 2.34 & 2.09 & 1.79 & 1.53 & 1.30 \\
\hline Miscellaneous & 0.12 & 0.10 & 0.09 & 0.08 & 0.06 \\
\hline Supervision \& Overhead & $\underline{0.18}$ & $\underline{0.16}$ & $\underline{0.14}$ & $\underline{0.12}$ & $\underline{0.10}$ \\
\hline Total Cost Year $\# 3^{\mathrm{b}}$ & $\overline{2.64}$ & $\overline{2.35}$ & $\overline{2.02}$ & $\overline{1.73}$ & $\overline{1.46}$ \\
\hline Total Three-Year Cumulative Costs & $\underline{\underline{28.71}}$ & $\underline{\underline{25.45}}$ & $\underline{\underline{22.36}}$ & 20.07 & $\underline{17.55}$ \\
\hline
\end{tabular}

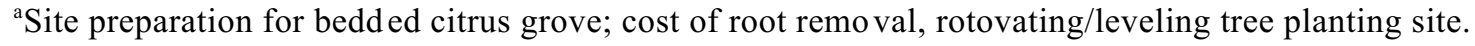

Fumigate planting site would cost approximately $\$ 2.50$ per tree.

${ }^{\mathrm{b}}$ Additional spray costs may be incurred if leafminer is a problem.

SOURCE: Ronald P. Muraro, Farm Management Economist, CREC, Lake Alfred, FL, December 2000. 
Table 13-A.-A listing of estimated comparative Central Florida (Ridge) citrus production costs per acre for $2002-2003^{\mathrm{z}}$

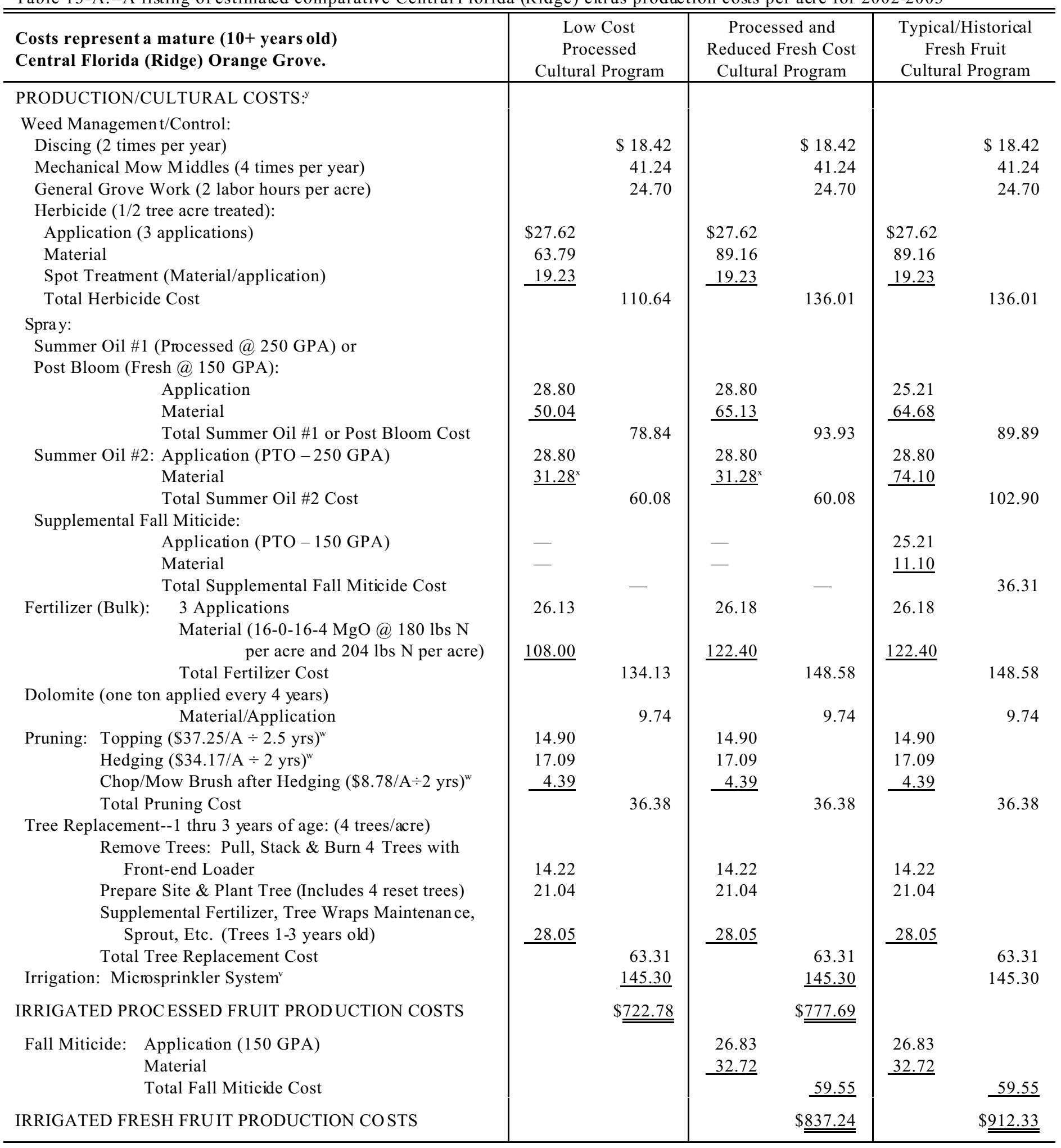

${ }^{\mathrm{z}}$ The listed estimated comparative costs are for the example grove situation described in the Economic Information Report Series entitled:

"Budgeting Costs and Returns for Central Florida Citrus Production" and may not represent your particular grove situation in Central Florida. 
Table 14-A.--Estimated average picking, roadsiding and hauling charges for Florida citrus, 2002-03

\begin{tabular}{|c|c|c|c|c|c|c|}
\hline & \multicolumn{3}{|c|}{ Fresh Fruit } & \multicolumn{3}{|c|}{ Processed Fruit } \\
\hline & & Range & Average & & Range & Average \\
\hline & & Box & $\$ /$ Box & & S/Box & $\$ /$ Box \\
\hline \multicolumn{7}{|l|}{ Picking Charges: } \\
\hline Early and Mid-Season Oranges & 0.72 & - 0.90 & 0.814 & 0.75 & - 0.90 & 0.806 \\
\hline Valencia Oranges & 0.79 & - $\quad 0.90$ & 0.835 & 0.76 & $-\quad 0.90$ & 0.810 \\
\hline Pink/Red Grapefruit & 0.60 & -0.80 & 0.692 & 0.55 & - $\quad 0.75$ & 0.625 \\
\hline White/Marsh Grapefruit & 0.60 & -0.77 & 0.668 & 0.50 & $-\quad 0.75$ & 0.600 \\
\hline Temples/Tangelos & 0.85 & - 0.90 & 0.883 & 0.85 & -0.90 & 0.860 \\
\hline \multirow[t]{4}{*}{ Tangerines } & 1.35 & -1.91 & 1.600 & & - & - \\
\hline & \multicolumn{3}{|c|}{ Fresh Fruit } & \multicolumn{3}{|c|}{ Processed Fruit } \\
\hline & \multicolumn{2}{|c|}{ Range } & Average & \multicolumn{2}{|c|}{ Range } & Average \\
\hline & \multicolumn{2}{|c|}{$\$ /$ Box } & \$/Box & \multicolumn{2}{|c|}{$\$ /$ Box } & $\$ /$ Box \\
\hline \multicolumn{7}{|l|}{ Roadsiding Charges: } \\
\hline Early and Mid-Season Oranges & 0.75 & -1.03 & 0.882 & 0.70 & -0.96 & 0.838 \\
\hline Valencia Oranges & 0.75 & -1.08 & 0.910 & 0.70 & -0.96 & 0.851 \\
\hline Pink/Red Grapefruit & 0.75 & -0.85 & 0.792 & 0.75 & -0.77 & 0.758 \\
\hline White/Marsh Grapefruit & 0.75 & - 0.81 & 0.778 & 0.75 & - 0.77 & 0.758 \\
\hline Temples/Tangelos & 0.90 & -0.95 & 0.927 & 0.76 & -0.90 & 0.850 \\
\hline \multirow[t]{4}{*}{ Tangerines } & 1.12 & -1.21 & 1.177 & & - & - \\
\hline & \multicolumn{3}{|c|}{ Fresh Fruit } & \multicolumn{3}{|c|}{ Processed Fruit } \\
\hline & \multicolumn{3}{|c|}{ All Varieties } & \multicolumn{3}{|c|}{ All Varieties } \\
\hline & \multicolumn{3}{|c|}{$\$ /$ Box } & \multicolumn{3}{|c|}{$\$ /$ Box } \\
\hline \multicolumn{7}{|l|}{ Hauling Charges: } \\
\hline 0 - 30 miles & \multicolumn{3}{|c|}{0.408} & \multicolumn{3}{|c|}{0.384} \\
\hline $31-50$ miles & \multicolumn{3}{|c|}{0.445} & \multicolumn{3}{|c|}{0.433} \\
\hline $51-80$ miles & \multicolumn{3}{|c|}{0.517} & \multicolumn{3}{|c|}{0.498} \\
\hline $81-100$ miles & \multicolumn{3}{|c|}{0.575} & \multicolumn{3}{|c|}{0.551} \\
\hline $100+$ miles & \multicolumn{3}{|c|}{0.663} & \multicolumn{3}{|c|}{0.625} \\
\hline
\end{tabular}


Table 15-A.-Estimated average packing charges for Florida citrus, 2002-03

\begin{tabular}{|c|c|c|c|c|c|}
\hline & $\begin{array}{l}\text { Domestic } \\
\text { Grapefruit }\end{array}$ & $\begin{array}{c}\text { Export } \\
\text { Grapefruit }\end{array}$ & Oranges & $\begin{array}{l}\text { Temples/ } \\
\text { Tangelos }\end{array}$ & Tangerines \\
\hline & & & $\$ /$ Carton & ---------- & -------- \\
\hline \multirow[t]{2}{*}{ Total Packing Charge ${ }^{a}$} & 3.544 & 3.992 & 3.731 & 4.214 & 4.733 \\
\hline & - & 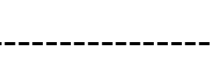 & $\$ /$ Box & $---\cdot$ & -------- \\
\hline Drenching Charge & 0.170 & 0.168 & 0.178 & 0.178 & 0.178 \\
\hline Packinghouse Elimination Charges & 0.566 & 0.588 & 0.600 & 0.600 & 0.633 \\
\hline Hauling Charge for Eliminations & 0.388 & 0.368 & 0.404 & 0.434 & 0.434 \\
\hline
\end{tabular}

${ }^{\text {a}}$ Total Packing Charge includes the following items:

1. Materials including mesh/plastic bags, labels/PLUs, etc.

2. Includes supervisor/foreman labor, grading, palletizing, shipping and general labor. Includes payroll taxes (FICA), workers' compensation, ground insurance, etc.

3. Other direct packing costs include: fruit treating; power, lights and water; repair maintenance; miscellaneous supplies; etc.

4. Indirect packing costs include such items as: insurance-fire and casualty; taxes and licenses, depreciation and rent.

5. G\&A costs include: office personnel (FICA, w/comp); packinghouse and general manager; office supplies; telephone; etc.

6. Special assessments include such items as: advertising taxes, inspection fees, Florida Citrus Packers; CAC.

NOTE: Packing charges represent a total of nine citrus packinghouses from both the Indian River and Interior production regions. 
31

Table 16-A.--Historic prices ${ }^{\mathrm{a}}$ for selected citrus varieties

\begin{tabular}{|c|c|c|c|c|c|c|c|}
\hline \multirow{3}{*}{ Crop year } & \multicolumn{7}{|c|}{ Variety } \\
\hline & \multirow{2}{*}{$\begin{array}{c}\text { Early and } \\
\text { mid }^{\mathrm{c}} \text {-season } \\
\text { oranges }\end{array}$} & \multirow{2}{*}{$\begin{array}{l}\text { Late season } \\
\text { oranges }^{\mathrm{d}}\end{array}$} & \multirow{2}{*}{$\begin{array}{l}\text { Temple } \\
\text { oranges }\end{array}$} & \multirow{2}{*}{$\begin{array}{c}\text { All } \\
\text { Tangerines }\end{array}$} & \multirow[t]{2}{*}{ Tangelos } & \multicolumn{2}{|c|}{ Seedless grapefruit ${ }^{\mathrm{e}}$} \\
\hline & & & & & & (white) & (colored) \\
\hline $1961-62$ & $\$ 1.93$ & $\$ 1.81$ & $\$ 2.17$ & $\$ 2.04$ & $\$ 3.36$ & $\$ 0.68$ & $\$ 0.86$ \\
\hline $1962-63$ & 2.17 & 3.50 & 3.09 & 3.02 & 4.66 & 1.29 & 1.81 \\
\hline $1963-64$ & 4.43 & 4.45 & 4.45 & 3.18 & 4.83 & 2.24 & 2.54 \\
\hline $1964-65$ & 2.57 & 2.28 & 2.77 & 2.68 & 4.00 & 1.51 & 1.82 \\
\hline $1965-66$ & 1.44 & 1.79 & 1.80 & 2.14 & 2.85 & 1.39 & 1.64 \\
\hline $1966-67$ & 0.81 & 1.08 & 0.88 & 1.06 & 1.64 & 0.73 & 0.94 \\
\hline $1967-68$ & 1.86 & 2.28 & 2.79 & 4.29 & 3.22 & 2.05 & 2.48 \\
\hline $1968-69$ & 1.56 & 1.83 & 2.22 & 2.55 & 2.47 & 0.98 & 1.15 \\
\hline $1969-70$ & 1.15 & 1.13 & 1.47 & 2.23 & 1.13 & 1.72 & 1.92 \\
\hline $1970-71$ & 1.10 & 1.91 & 1.91 & 1.88 & 1.04 & 1.89 & 2.15 \\
\hline $1971-72$ & 1.98 & 2.11 & 1.95 & 2.97 & 1.69 & 2.27 & 2.69 \\
\hline $1972-73$ & 1.43 & 1.71 & 1.95 & 2.37 & 1.39 & 2.06 & 2.53 \\
\hline $1973-74$ & 1.38 & 1.59 & 1.64 & 2.82 & 1.25 & 1.58 & 2.12 \\
\hline $1974-75$ & 1.46 & 1.82 & 1.68 & 3.05 & 1.45 & 1.55 & 2.59 \\
\hline $1975-76$ & 1.69 & 1.88 & 1.79 & 3.02 & 1.42 & 1.29 & 2.23 \\
\hline $1976-77$ & 1.89 & 2.63 & 2.16 & 3.29 & 1.42 & 1.49 & 2.04 \\
\hline $1977-78$ & 3.90 & 4.40 & 3.92 & 4.79 & 3.29 & 1.47 & 2.09 \\
\hline $1978-79$ & 4.44 & 4.95 & 4.89 & 4.99 & 3.90 & 2.21 & 3.13 \\
\hline $1979-80$ & 3.59 & 3.89 & 2.89 & 4.25 & 2.87 & 3.12 & 3.80 \\
\hline $1980-81$ & 3.67 & 4.63 & 4.21 & 5.45 & 3.92 & 3.46 & 4.22 \\
\hline $1981-82$ & 4.27 & 4.29 & 4.01 & 6.23 & 3.58 & 1.92 & 2.80 \\
\hline $1982-83$ & 4.88 & 5.41 & 3.99 & 7.57 & 4.37 & 1.51 & 3.20 \\
\hline $1983-84$ & 5.09 & 6.72 & 5.34 & 5.93 & 4.28 & 2.08 & 4.05 \\
\hline $1984-85$ & 7.30 & 6.88 & 5.59 & 15.91 & 7.08 & 3.02 & 4.84 \\
\hline $1985-86$ & 3.92 & 3.97 & 3.01 & 12.69 & 4.06 & 3.56 & 4.98 \\
\hline $1986-87$ & 4.56 & 6.02 & 3.60 & 10.92 & 3.72 & 4.45 & 5.80 \\
\hline $1987-88$ & 6.72 & 8.73 & 5.69 & 12.99 & 5.58 & 5.35 & 5.93 \\
\hline $1988-89$ & 6.63 & 8.41 & 5.46 & 12.64 & 6.31 & 4.33 & 4.71 \\
\hline $1989-90$ & 6.01 & 6.53 & 5.64 & 15.28 & 5.10 & 5.21 & 6.30 \\
\hline $1990-91$ & 5.38 & 6.58 & 6.31 & 17.10 & 6.11 & 4.59 & 6.85 \\
\hline $1991-92$ & 5.44 & 6.65 & 6.51 & 18.00 & 7.16 & 6.46 & 6.87 \\
\hline $1992-93$ & 3.23 & 3.88 & 2.99 & 13.75 & 3.31 & 2.22 & 3.11 \\
\hline 1993-94 & 3.76 & 4.61 & 2.73 & 9.83 & 2.38 & 3.23 & 3.38 \\
\hline $1994-95$ & 3.25 & 4.41 & 3.47 & 11.98 & 2.64 & 2.58 & 1.66 \\
\hline $1995-96$ & 3.62 & 5.57 & 4.44 & 12.59 & 3.63 & 2.14 & 1.77 \\
\hline 1996-97 & 3.18 & 4.07 & 3.22 & 7.99 & 2.19 & 1.12 & 1.91 \\
\hline $1997-98$ & 2.81 & 4.88 & 3.07 & 8.49 & 1.66 & 0.93 & 1.50 \\
\hline $1998-99$ & 4.35 & 5.58 & 5.12 & 12.07 & 4.53 & 1.95 & 2.65 \\
\hline 1999-00 & 3.19 & 4.33 & 2.55 & 6.67 & 2.52 & 3.87 & 3.36 \\
\hline $2000-01$ & 2.60 & 4.02 & 2.05 & 6.40 & 1.27 & 2.07 & 2.28 \\
\hline $2001-02^{\mathrm{f}}$ & 2.46 & 3.99 & 2.28 & 7.69 & 2.37 & 1.95 & 2.17 \\
\hline
\end{tabular}

${ }^{a}$ On-tree average price per box (1-3/5 bushel box equivalent) for all methods of sale minus pick and haul charges.

${ }^{b}$ Navel and Hamlin $\quad$ 'Parson Brown and Pineapple $\quad{ }^{\mathrm{d}}$ Valencia $\quad{ }^{\mathrm{e}}$ Marsh (white) or pink $\quad{ }^{\mathrm{f}}$ Preliminary Source: Florida Agricultural Statistics Service. 
Table 17-A.--Debt which can be supported per $\$ 1,000.00$ annual paym ent capacity

\begin{tabular}{|c|c|c|c|c|c|c|c|c|c|c|c|c|c|c|c|}
\hline \multirow{2}{*}{$\begin{array}{c}\text { Loan } \\
\text { term } \\
\text { (years) }\end{array}$} & \multicolumn{15}{|c|}{ Interest rate paid on the loan } \\
\hline & $8.0 \%$ & $8.5 \%$ & $9.0 \%$ & $9.5 \%$ & $10.0 \%$ & $10.5 \%$ & $11.0 \%$ & $11.5 \%$ & $12.0 \%$ & $12.5 \%$ & $13.0 \%$ & $13.5 \%$ & $14.0 \%$ & $14.5 \%$ & $15.0 \%$ \\
\hline 1 & 926 & 922 & 917 & 913 & 909 & 905 & 901 & 897 & 893 & 889 & 885 & 881 & 877 & 873 & 870 \\
\hline 2 & 1,783 & 1,771 & 1,759 & 1,747 & 1,754 & 1,724 & 1,713 & 1,701 & 1,690 & 1,679 & 1,668 & 1,657 & 1,647 & 1,636 & 1,626 \\
\hline 3 & 2,577 & 2,554 & 2,531 & 2,509 & 2,487 & 2,465 & 2,444 & 2,423 & 2,402 & 2,381 & 2,361 & 2,341 & 2,322 & 2,302 & 2,283 \\
\hline 4 & 3,312 & 3,276 & 3,240 & 3,204 & 3,170 & 3,136 & 3,102 & 3,070 & 3,037 & 3,006 & 2,974 & 2,944 & 2,914 & 2,884 & 2,855 \\
\hline 5 & 3,993 & 3,941 & 3,890 & 3,840 & 3,791 & 3,743 & 3,696 & 3,650 & 3,605 & 3,561 & 3,517 & 3,475 & 3,433 & 3,392 & 3,352 \\
\hline 6 & 4,623 & 4,554 & 4,486 & 4,420 & 4,355 & 4,292 & 4,230 & 4,170 & 4,111 & 4,054 & 3,998 & 3,942 & 3,889 & 3,836 & 3,784 \\
\hline 7 & 5,206 & 5,119 & 5,033 & 4,950 & 4,868 & 4,789 & 4,712 & 4,640 & 4,564 & 4,492 & 4,423 & 4,355 & 4,288 & 4,224 & 4,160 \\
\hline 8 & 5,747 & 5,639 & 5,535 & 5,433 & 5,335 & 5,239 & 5,146 & 5,056 & 4,968 & 4,882 & 4,799 & 4,718 & 4,639 & 4,562 & 4,487 \\
\hline 9 & 6,247 & 6,119 & 5,995 & 5,875 & 5,759 & 5,646 & 5,537 & 5,431 & 5,328 & 5,228 & 5,132 & 5,038 & 4,946 & 4,858 & 4,772 \\
\hline 10 & 6,710 & 6,561 & 6,418 & 6,279 & 6,145 & 6,015 & 5,889 & 5,768 & 5,650 & 5,536 & 5,426 & 5,319 & 5,216 & 5,116 & 5,019 \\
\hline 11 & 7,139 & 6,969 & 6,805 & 6,647 & 6,495 & 6,348 & 6,207 & 6,070 & 5,938 & 5,810 & 5,687 & 5,568 & 5,453 & 5,341 & 5,234 \\
\hline 12 & 7,536 & 7,345 & 7,161 & 6,984 & 6,814 & 6,650 & 6,492 & 6,341 & 6,194 & 6,054 & 5,918 & 5,787 & 5,660 & 5,538 & 5,421 \\
\hline 13 & 7,904 & 7,691 & 7,487 & 7,291 & 7,103 & 6,923 & 6,750 & 6,583 & 6,424 & 6,270 & 6,122 & 5,979 & 5,842 & 5,710 & 5,583 \\
\hline 14 & 8,244 & 8,010 & 7,786 & 7,572 & 7,367 & 7,170 & 6,982 & 6,801 & 6,628 & 6,462 & 6,302 & 6,149 & 6,002 & 5,861 & 5,724 \\
\hline$\underline{15}$ & 8,559 & 8,304 & 8,061 & 7,828 & 7,606 & 7,394 & 7,191 & $\underline{6,997}^{\mathrm{a}}$ & 6,811 & 6,633 & 6,462 & 6,299 & 6,142 & 5,992 & 5,847 \\
\hline 16 & 8,851 & 8,576 & 8,313 & 8,062 & 7,824 & 7,596 & 7,379 & 7,172 & 6,974 & 6,785 & 6,604 & 6,431 & 6,265 & 6,106 & 5,954 \\
\hline 17 & 9,122 & 8,825 & 8,543 & 8,276 & 8,022 & 7,779 & 7,549 & 7,329 & 7,119 & 6,920 & 6,729 & 6,547 & 6,373 & 6,207 & 6,048 \\
\hline 18 & 9,372 & 9,056 & 8,756 & 8,471 & 8,201 & 7,945 & 7,702 & 7,470 & 7,250 & 7,040 & 6,840 & 6,649 & 6,467 & 6,294 & 6,128 \\
\hline 19 & 9,603 & 9,268 & 8,950 & 8,650 & 8,365 & 8,095 & 7,839 & 7,596 & 7,366 & 7,146 & 6,938 & 6,739 & 6,551 & 6,370 & 6,198 \\
\hline$\underline{20}$ & 9,818 & 9,463 & 9,129 & 8,812 & 8,514 & 8,231 & 7,963 & $\underline{7,710^{\mathrm{a}}}$ & 7,469 & 7,241 & 7,025 & 6,819 & 6,623 & 6,437 & 6,259 \\
\hline 25 & 10,675 & 10,234 & 9,823 & 9,438 & 9,077 & 8,739 & 8,422 & $\overline{8,123}$ & 7,843 & 7,579 & 7,330 & 7,095 & 6,873 & 6,663 & 6,464 \\
\hline 30 & 11,258 & 10,747 & 10,274 & 9,835 & 9,427 & 9,047 & 8,868 & 8,364 & 8,055 & 7,766 & 7,496 & 7,242 & 7,003 & 6,778 & 6,566 \\
\hline 35 & 11,655 & 11,088 & 10,567 & 10,087 & 9,644 & 9,234 & 8,855 & 8,503 & 8,175 & 7,870 & 7,586 & 7,320 & 7,070 & 6,836 & 6,617 \\
\hline 40 & 11,925 & 11,315 & 10,757 & 10,247 & 9,779 & 9,348 & 8,951 & 8,587 & 8,244 & 7,928 & 7,634 & 7,361 & 7,105 & 6,866 & 6,642 \\
\hline
\end{tabular}

${ }^{a}$ Example. Assumes a $\$ 10,000$ after tax income at $11.5 \%$ interest rate and a 15 -year term mortgage, the total debt which can be supported is $\$ 69,970$ $(\$ 6,997 \times 10)$. At $11.5 \%$ interest rate and a 20 -year term mortgage, the total debt which can be supported is $\$ 77,100(\$ 7,710 \times 10)$. 